\title{
A Survey of Parametrized Variational Principles and Applications to Computational Mechanics
}

\author{
Carlos A. Felippa \\ Department of Aerospace Engineering Sciences and \\ Center for Space Structures and Controls \\ University of Colorado \\ Boulder, Colorado 80309-0429, USA
}

September 1992

Revised May 1993

Report No. CU-CSSC-92-11

Written version of an invited presentation to the First International Mechanics

Seminar held at the Institute de Mécanique, Grenoble, France, May 1992.

Accepted for publication in Computer Methods in Applied Mechanics and Engineering

Research supported by NASA Langley Research Center under Grant NAS1-756, monitored by Dr. J. Housner. 


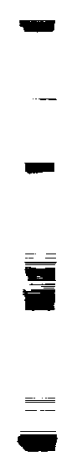

$=$
$\bar{E}$
$=$

$\underline{\underline{\underline{\underline{\underline{E}}}}}$

$=$

$-$

- 


\title{
A SURVEY OF PARAMETRIZED VARIATIONAL PRINCIPLES AND APPLICATIONS TO COMPUTATIONAL MECHANICS
}

\author{
Carlos A. Felippa \\ Department of Aerospace Engineering Sciences and \\ Center for Space Structures and Controls \\ University of Colorado, Boulder \\ Boulder, CO 80309-0429, USA
}

\begin{abstract}
This survey paper describes recent developments in the area of parametrized variational principles (PVPs) and selected applications to finite-element computational mechanics. A PVP is a variational principle containing free parameters that have no effect on the Euler-Lagrange equations. The theory of single-field PVPs, based on gauge functions (also known as null Lagrangians) is a subset of the Inverse Problem of Variational Calculus that has limited value. On the other hand, multifield PVPs are more interesting from theoretical and practical standpoints. Following a tutorial introduction, the paper describes the recent construction of multifield PVPs in several areas of elasticity and electromagnetics. It then discusses three applications to finite-element computational mechanics: the derivation of high-performance finite elements, the development of element-level error indicators, and the construction of finite element templates. The paper concludes with an overview of open research areas.
\end{abstract}




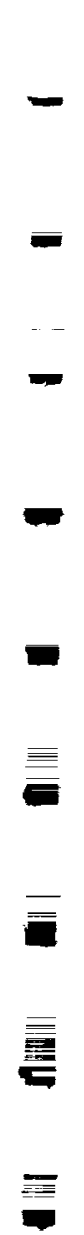

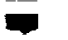

$=$

产

$\overline{\bar{\equiv}}$

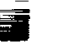

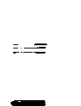

단

$\bar{E}$

-

$\bar{\equiv}$

$-$

= 


\section{INTRODUCTION}

This paper is the written version of an invited presentation to the First International Seminar in Mechanics (SIMG) held at the Institute de Mécanique de Grenoble on May 1992. It reviews the recent development and selected applications of parametrized variational principles (PVPs) in mechanics.

A PVP derives from a functional with free parameters if the Euler-Lagrange equations turn out to be independent of those parameters. In this survey a sharp distinction is made between single-field PVPs, in which only one primary field is varied, and multifield (mixed) PVPs. Single-field PVPs are well known but lack practical importance. They are used here only in the tutorial introduction of Section 3. Multifield PVPs are less understood but far more interesting from the standpoints of theory and applications.

The study of multifield PVPs was originally motivated by the desire of finding a variational basis for some high-performance finite elements, as outlined in Section 5.6. As sometimes happens, this modest goal led to unexpected discoveries, chief among them a general parametrization of the functionals of classical elasticity. Subsequently more applications to finite elements were revealed.

The paper is organized as follows. Section 2 is philosophical in nature and speculates on the potential impact of this emerging area. Section 3 is a tutorial that presents basic concepts and definitions using single-field PVPs. Section 4 introduces multifield PVPs. Sections 5 through 8 outline the construction of PVPs for several application areas, with emphasis on linear elasticity. Those readers with interest restricted to theoretical aspects may skip Sections 9 through 12, which present applications of PVPs to finite elements in computational mechanics. Of these the first application: development of high-performance elements, is the most developed one. The other applications: error estimation and templates, are in an exploratory stage and rely largely on conjectures and numerical experimentation. The paper concludes with an overview of topics that may deserve further research.

\section{PHILOSOPHICAL DIGRESSION}

Necessity is the mother of invention. Although this aphorism is normally applied to technological and industrial advances, there have been instances of its validity in mathematical physics. Three examples can be cited.

Operational Calculus. Invented by Oliver Heaviside to solve the ODEs and PDEs brought forth by the initial applications of Maxwell's electromagnetic theory in the 1880s. Heaviside's calculus was based on heuristic rules, most of which were eventually justified by the development of transform theory and associated axiomatics. By now operational calculus is a standard branch of applied and theoretical mathematics.

Delta Calculus. Although the underlying ideas existed in latent form prior to 1920, delta functions were popularized by Paul Dirac as a tool to formulate one of the three versions 
of the "old" quantum mechanics. Dirac's recipes were eventually justified twenty years later by the theory of distributions and generalized functions. Delta calculus is presently an important analytical and computational tool for engineers and scientists.

Finite Element Method. The pioneering 1943 paper by Courant [15] attracted no attention then because no need was addressed and digital computers had not arrived. The rapid development of the FEM in the aerospace industry can be traced back to efforts in the early $1950 \mathrm{~s}$ to formulate a satisfactory computer-based procedure for the stiffness analysis of sweptback, fixed delta wings [61]. This involved an extension to continua of by-then established discrete-element procedures collectively known as "matrix structural analysis" $[29,34,35,36]$, which had culminated in the elegant unification of Argyris and Kelsey [3]. The method was named in 1960 by Clough [14]. In this early period primary concern was given to matching experimental results rather than to the underlying theory. The connection with variational principles and approximation theory was established in the 1960s after the FEM proved successful on a much wider class of problems.

A common thread runs through these examples:

1. An urgent application need is established.

2. Response to need produces an unpolished tool.

3. Tool is recognized as useful beyond its initial purpose.

4. With interest from mathematicians aroused, theory (and eventually axiomatization) follows.

As regards parametrized variational principles, only the first two steps have been verified. The motivating need was the justification of high-performance finite elements. Although there are indications of usefulness beyond that context, such applications remains to be firmly established. In any event, much theory is lacking.

\section{PARAMETRIZED VARIATIONAL PRINCIPLES: CONCEPT}

\subsection{A Single-Field Example}

Let $u=u(x) \in \mathcal{C}^{2}[a, b]$ be a real-valued function, while $\beta$ is a free parameter. Consider the parametrized functional

$$
\Pi(u ; \beta)=\frac{1}{2} \int_{a}^{b} F(u ; \beta) d x=\frac{1}{2} \int_{a}^{b}\left[-\left(u^{\prime}\right)^{2}+2 \beta u u^{\prime}+u^{2}\right] d x,
$$

where ()$^{\prime} \equiv \partial() / \partial x$. The Euler-Lagrange equation associated with $\delta \Pi=0$ is

$$
E(u)=u^{\prime \prime}+u=0
$$

which is independent of $\beta$. The statement $\delta \Pi=0$ will be called a parametrized variational principle, or PVP. Notice, however, that the natural boundary conditions

$$
F_{u^{\prime}}(a)=\beta u(a)-u^{\prime}(a)=0, \quad F_{u^{\prime}}(b)=\beta u(b)-u^{\prime}(b)=0,
$$


are not independent of $\beta$ unless $u(a)=u(b)=0$.

This example can be easily generalized. Chose an arbitrary function $Q(u, x) \in \mathcal{C}^{2}[a, b]$ and consider

$$
\Pi(u ; \beta)=\frac{1}{2} \int_{a}^{b}\left[-\left(u^{\prime}\right)^{2}+2 \beta Q u^{\prime}+u^{2}\right] d x
$$

The Euler-Lagrange equation for (4) is gain (2) - the simpler form (1) is in fact obtained if $Q=u$. Another generalization (noted by a reviewer) is

$$
\Pi(u ; \beta)=\frac{1}{2} \int_{a}^{b}\left[-\left(u^{(m)}\right)^{2}+2 \beta u^{(m)} u^{(m-1)}+\left(u^{(m-1)}\right)^{2}\right] d x, \quad u^{(j)} \equiv d^{m} u / d u^{m},
$$

for $m>0$, whose Euler-Lagrange equation is $u^{(m+2)}+u^{(m)}=0$. Despite their simplicity these functionals have engineering significance; e.g. the suitably normalized (5) with $m=2$ governs the buckling of an Euler-Bernoulli beam.

\subsection{Principal and Gauge Functionals}

The explanation for the behavior of the preceding functionals is straightforward. Consider for simplicity (2) in which the parametrized term is separated as

$$
\begin{gathered}
\Pi(u ; \beta)=\Pi_{P}(u)+\beta \Pi_{G}(u), \\
\Pi_{P}(u)=\frac{1}{2} \int_{a}^{b}\left[-\left(u^{\prime}\right)^{2}+u^{2}\right] d x, \quad \Pi_{G}(u)=\int_{a}^{b} u u^{\prime} d x=\frac{1}{2}\left[u^{2}(b)-u^{2}(a)\right] .
\end{gathered}
$$

Here $\Pi_{P}$ is the principal functional whereas $\Pi_{G}$ is a gauge functional. The latter is also called a null Lagrangian in the literature. Because $\Pi_{G}$ depends only on boundary values, its contribution to the Euler-Lagrange equation of $\Pi$ is obviously zero thus cancelling the effect of $\beta$.

The converse statement is also a well known theorem of variational calculus: if the EulerLagrange equation of a single-field functional vanishes identically, that functional must depend only on boundary values. See, for example, Section 1.18 of Fox [30].

The second example (5) illustrates the addition of an arbitrary one-dimensional gauge function. Should the single-field functional depend on several independent variables $x, y, \ldots$, one can obviously add the divergence or rotor of multidimensional functions multiplied by free parameters, because application of Green's or Stokes' theorems reduces such functions to boundary terms.

\subsection{Terminology}

A functional that contains one or more free parameters, such as $\Pi$ in the foregoing example, is called a parametrized functional. If its Euler-Lagrange equation is independent of the parameters, the stationarity condition $\delta \Pi=0$ is called a parametrized variational principle or PVP. 


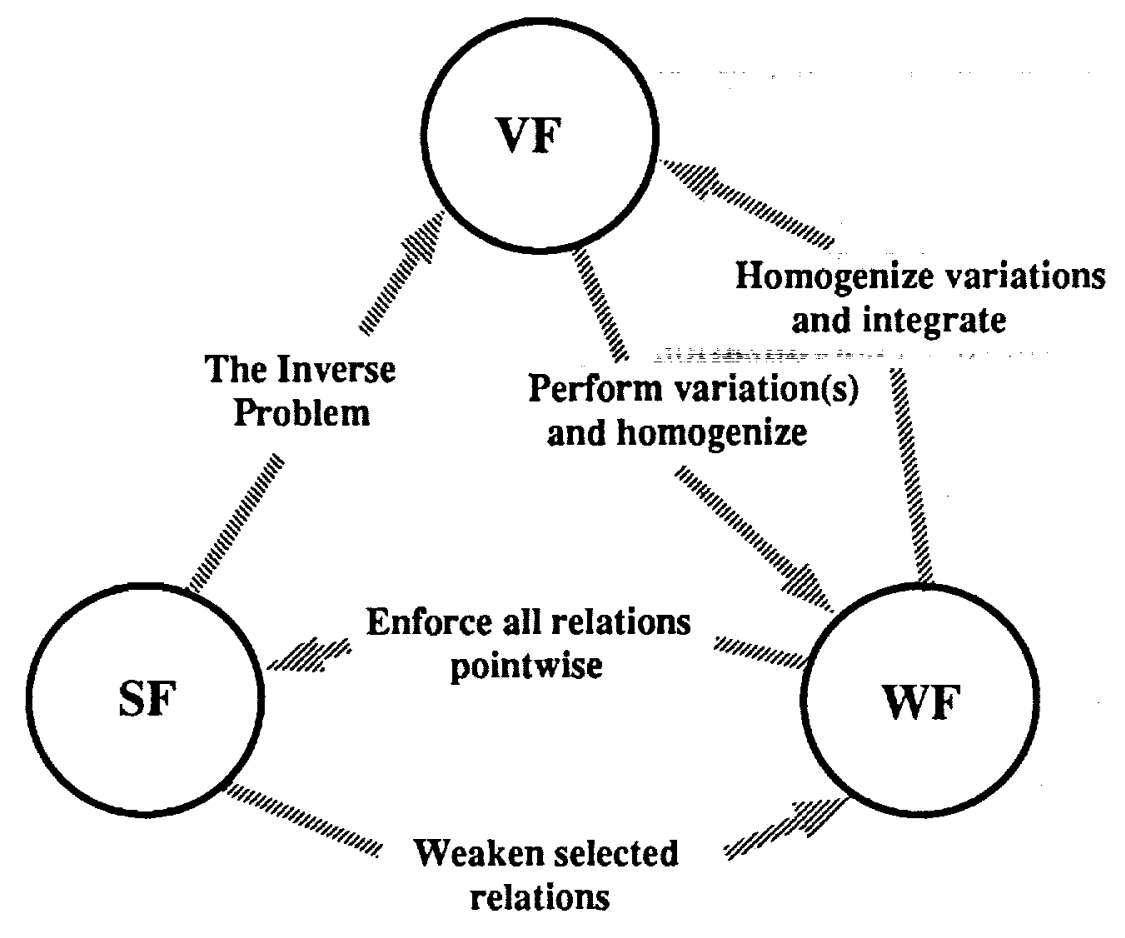

Fig. 1. Diagram sketching Strong, Weak and Variational Forms, and relationships between form pairs. (Weak Forms are also called weighted-residual equations, variational equations, Galerkin equations and integral statements in the literature.)

A PVP is most useful from the standpoint of applications if the value of the functional, evaluated at an extremal, is independent of the free parameters. This value has often the meaning of energy. Such a principle will be called an invariant parametrized variational principle or IPVP. The example functional (3) yields an IPVP if $\left[u^{\prime \prime}(b)\right]^{2}=\left[u^{\prime \prime}(a)\right]^{2}$ or if $\left[u^{\prime}(b)\right]^{2}=\left[u^{\prime}(a)\right]^{2}$. (To prove the latter, insert $u=-u^{\prime \prime}$ into $\Pi_{G}$ and integrate.) If $[u(b)]^{2}=[u(a)]^{2}, \Pi$ is independent of $\beta$ for any $u(x)$, not just an extremal. This will be called an absolutely invariant PVP, or AIPVP.

The most useful IPVPs and AIPVPs, however, are those in which invariance does not depend on boundary conditions. Such functionals will be encountered in following sections.

\subsection{Connections with the Inverse Problem}

The study of single-field PVP's constitutes a particular topic of the Inverse Problem of Variational Calculus: given a system of differential equations - herein called the Strong Form or SF - find the Lagrangians that have that system as Euler-Lagrange equations. These Lagrangians (if they exist) collectively embody the Variational Form (VF) of the problem. The Weak Form (WF) of the problem, which is also known by the alternative names listed in Figure 1, is an intermediary between SF and VF. Relations between SF, $\mathrm{WF}$ and VF are annotated in Figure 1. 
The Inverse Problem linking ordinary differential equations to single-field functionals is treated in several monographs $[55,57,62]$. On the other hand, the multifield case is much less developed. Aside from the developments presented here, parametrized functionals have also been studied by the Beijing school $[37,38]$ but without establishing connections to the Finite Element Method.

\section{MULTIFIELD VARIATIONAL PRINCIPLES}

Single-field PVP have limited practical and theoretical value. After all, gauge functions have been known for a long time but never attracted much attention. Multifield (mixed) PVPs are more interesting and fruitful, both theoretically and practically. In this section two examples are worked out to unveil the flavor of the more complicated cases and expose the reader to the notational conventions used in following sections.

\subsection{One-Dimensional Example}

As our first encounter with a multifield PVP, the following 6-coefficient generalization of (2) to two independently varied fields: $u$ and $p=u^{\prime}$, is postulated:

$$
\Pi(u, p ; \mathbf{J})=\frac{1}{2} \int_{a}^{b}\left\{\begin{array}{c}
u \\
u^{\prime} \\
p
\end{array}\right\}^{T}\left[\begin{array}{lll}
j_{11} & j_{12} & j_{13} \\
j_{12} & j_{22} & j_{23} \\
j_{13} & j_{23} & j_{33}
\end{array}\right]\left\{\begin{array}{c}
u \\
u^{\prime} \\
p
\end{array}\right\} d x=\frac{1}{2} \int_{a}^{b} \mathbf{z}^{T} \mathbf{J z} d x
$$

in which

$$
\mathbf{z}=\left\{\begin{array}{c}
u \\
u^{\prime} \\
p
\end{array}\right\}, \quad \mathbf{J}=\left[\begin{array}{lll}
j_{11} & j_{12} & j_{13} \\
j_{12} & j_{22} & j_{23} \\
j_{13} & j_{23} & j_{33}
\end{array}\right]
$$

are the generalized field vector and the functional generating matrix, respectively. This parameter matrix - the "kernel" of the quadratic form in the $\mathbf{z}$ vector - may be taken as symmetric because only its symmetric part participates in the first variation. This general notational arrangement is followed in more complicated linear problems of mathematical physics presented later.

The Euler-Lagrange equations for $\delta_{u} \Pi=0$ and $\delta_{p} \Pi=0$ are

$$
\begin{array}{ll}
E_{u}: & j_{11} u+j_{12} u^{\prime}+j_{13} p-\left(j_{12} u^{\prime}+j_{22} u^{\prime \prime}+j_{23}\right) p^{\prime}=0, \\
E_{p}: & j_{13} u+j_{23} u^{\prime}+j_{33} p=0 .
\end{array}
$$

Consistency of (10) with the field equations $u^{\prime}-p=0$ and $u+p^{\prime}=0$ dictates that $\mathbf{J}$ be of the form

$$
\mathbf{J}=\left[\begin{array}{ccc}
\alpha & \beta & 0 \\
\beta & 0 & -\alpha \\
0 & -\alpha & \alpha
\end{array}\right]
$$

Thus the functional is found to depend on two independent free parameters: $\alpha$ and $\beta$. Is this PVP invariant? At an extremal, $p=u^{\prime}$, which replaced into (8) yields $\Pi=$ $\frac{1}{2} \int_{a}^{b}\left(\alpha u^{2}-\beta u^{\prime 2}\right) d x$. Therefore this is not generally an IPVP. 
A more satisfying parametrization that automatically verifies invariance is obtained by taking three independent fields, as in the example that follows.

\subsection{A PDE Example: 2-D Isotropic Poisson's Equation}

As second example we consider the isotropic Poisson's equation (the Laplace equation with a source term) posed over a finite two-dimensional region $\Omega$ bounded by a curve $\Gamma$ :

$$
k \nabla^{2} u=k\left(\frac{\partial^{2} u}{\partial x^{2}}+\frac{\partial^{2} u}{\partial y^{2}}\right)=-f \quad \text { in } \Omega,
$$

where $u=u(x, y)$ is the unknown function, $k(x, y)>0$ and $f(x, y)$ are given scalar functions in $\Omega$. Boundary $\Gamma$ is decomposed into $\Gamma_{u} \cup \Gamma_{q}$, on which Dirichlet and Neumann (flux-type) boundary conditions, respectively, are imposed:

$$
u=\widehat{u} \quad \text { on } \quad \Gamma_{u}, \quad q=k \frac{\partial u}{\partial n}=k(\operatorname{grad} u)^{T} \mathbf{n}=\widehat{q} \quad \text { on } \quad \Gamma_{q},
$$

where $\widehat{u}$ and $\widehat{q}$ are prescribed on $\Gamma_{u}$ and $\Gamma_{q}$, respectively, and $\mathbf{n}$ is the exterior unit normal on $\Gamma$. The single-field functional $\Pi(u)$ associated with (12) and (13) is well known:

$\Pi=U(u)-P(u), \quad U(u)=\frac{1}{2} \int_{\Omega} k\left[\left(\frac{\partial u}{\partial x}\right)^{2}+\left(\frac{\partial u}{\partial y}\right)^{2}\right] d \Omega, P(u)=\int_{\Omega} f u d \Omega+\int_{\Gamma_{q}} \hat{q} u d \Gamma$.

Here $U(u)$ has the meaning of internal energy whereas $P(u)$ is an external energy associated with the source and prescribed-flux terms. As noted in Section 3.2, functional (14) can be trivially parametrized by adding multiples of the divergence or rotor of gauge functions. But such PVPs have no practical importance.

To begin the construction of a multifield PVP we introduce the two intermediate vector fields: gradient $\mathbf{g}$ and flux vector $\mathbf{p}$, as candidates for independent variation:

$$
\mathbf{g}=\left\{\begin{array}{l}
g_{x} \\
g_{y}
\end{array}\right\}=\operatorname{grad} u=\left\{\begin{array}{l}
\partial u / \partial x \\
\partial u / \partial y
\end{array}\right\}, \quad \mathbf{p}=\left\{\begin{array}{l}
p_{x} \\
p_{y}
\end{array}\right\}=k \mathbf{g}=k \operatorname{grad} u=k\left\{\begin{array}{l}
\partial u / \partial x \\
\partial u / \partial y
\end{array}\right\}
$$

The PDE (12) decomposes into the three field equations

$$
\mathbf{g}=\nabla u=\operatorname{grad} u, \quad \mathbf{p}=k \mathbf{g}, \quad \nabla^{T} \mathbf{p}+f=\operatorname{div} \mathbf{p}+f=0,
$$

where div $\equiv \nabla^{T}$ is the divergence operator. In mechanical applications these are called the kinematic, constitutive and balance equations, respectively.

The three field equations (16) and two boundary conditions (13) collectively make up the Strong Form (SF) of the isotropic Poisson's equation. This SF is graphically represented in Figure 2 using a modified Tonti diagram. Relations such as $\mathbf{g}=\operatorname{grad} u$ are called strong connections (which means that they are enforced point by point) and depicted as solid lines. The main departure of Figure 2 from Tonti's original diagrams $[47,59,60]$ is the explicit separation of field equations and boundary conditions; this has been found useful in teaching variational methods. In addition, a graphical distinction is made between unknown and data fields, as indicated in Figure 2, also for instructional reasons. 


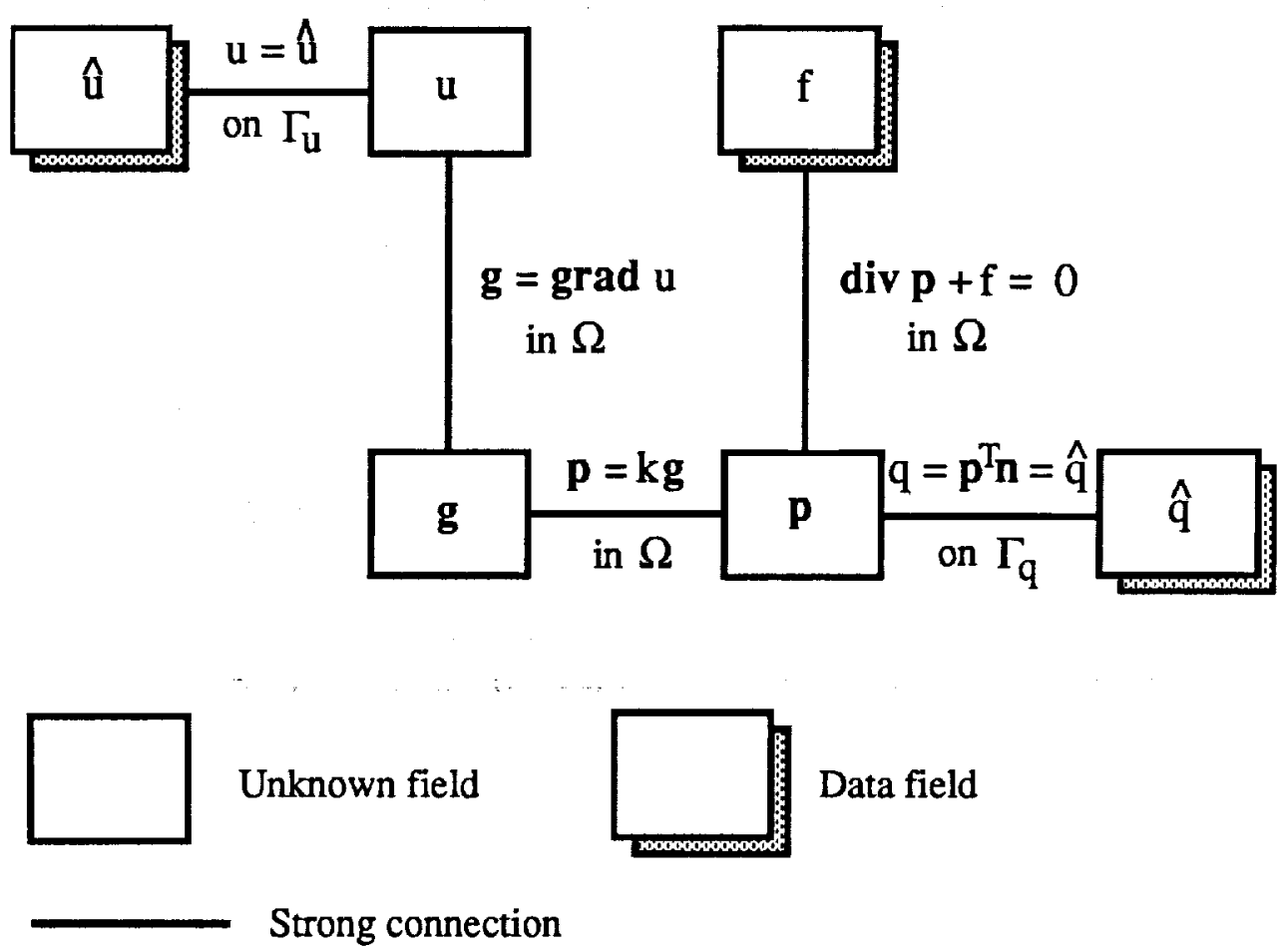

Fig. 2. Graphical representation of the Strong Form (SF) of the isotropic two-dimensional Poisson's equation (12) with the B.C.s (13) as a modified Tonti diagram.

\subsection{A 3-Field PVP for Poisson's Equation}

The configuration of (14) suggests trying to parametrize the internal energy $U$ as a threefield quadratic form, leading to the arrangement displayed below in full:

$$
U(u, \mathbf{g}, \mathbf{p})=\frac{1}{2} \int_{\Omega}\left\{\begin{array}{c}
p_{x} \\
p_{y} \\
k g_{x} \\
k g_{y} \\
k \partial u / \partial x \\
k \partial u / \partial y
\end{array}\right\}\left[\begin{array}{llllll}
j_{11} & j_{11} & j_{12} & j_{12} & j_{13} & j_{13} \\
j_{11} & j_{11} & j_{12} & j_{12} & j_{13} & j_{13} \\
j_{12} & j_{12} & j_{22} & j_{22} & j_{23} & j_{23} \\
j_{12} & j_{12} & j_{22} & j_{22} & j_{23} & j_{23} \\
j_{13} & j_{13} & j_{23} & j_{23} & j_{33} & j_{33} \\
j_{13} & j_{13} & j_{23} & j_{23} & j_{33} & j_{33}
\end{array}\right]\left\{\begin{array}{c}
k^{-1} p_{x} \\
k^{-1} p_{y} \\
g_{x} \\
g_{y} \\
\partial u / \partial x \\
\partial u / \partial y
\end{array}\right\} d \Omega
$$

in which for simplicity the explicit dependence of $U$ on the $j$ coefficients is dropped from its arguments. The symmetry of the kernel matrix can be justified as in the previous example, whereas its $2 \times 2$ block structure is a consequence of avoiding distortions in the vector-component contributions to the internal energy. This functional is fully specified if the $3 \times 3$ generating matrix $\mathbf{J}$, which has the same form of (9), is given.

Now (17) looks unduly complicated for such a simple problem. At the same time, what is being varied is not easily seen. We clarify and simplify this form in two steps: passing 
to matrix-vector notation, and then applying the primary-versus derived-field convention explained below:

$$
\begin{aligned}
U(\tilde{u}, \tilde{\mathbf{g}}, \tilde{\mathbf{p}}) & =\frac{1}{2} \int_{\Omega}\left\{\begin{array}{c}
\tilde{\mathbf{p}} \\
k \tilde{\mathbf{g}} \\
k \operatorname{grad} \tilde{u}
\end{array}\right\}^{T}\left[\begin{array}{lll}
j_{11} \mathbf{I} & j_{12} \mathbf{I} & j_{13} \mathbf{I} \\
j_{12} \mathbf{I} & j_{22} \mathbf{I} & j_{23} \mathbf{I} \\
j_{13} \mathbf{I} & j_{23} \mathbf{I} & j_{33} \mathbf{I}
\end{array}\right]\left\{\begin{array}{c}
k^{-1} \tilde{\mathbf{p}} \\
\tilde{\mathbf{g}} \\
\operatorname{grad} \tilde{u}
\end{array}\right\} d \Omega \\
& =\frac{1}{2} \int_{\Omega}\left\{\begin{array}{c}
\tilde{\mathbf{p}} \\
\mathbf{p}^{g} \\
\mathbf{p}^{u}
\end{array}\right\}\left[\begin{array}{lll}
j_{11} \mathbf{I} & j_{12} \mathbf{I} & j_{13} \mathbf{I} \\
j_{12} \mathbf{I} & j_{22} \mathbf{I} & j_{23} \mathbf{I} \\
j_{13} \mathbf{I} & j_{23} \mathbf{I} & j_{33} \mathbf{I}
\end{array}\right]\left\{\begin{array}{c}
\mathbf{g}^{p} \\
\tilde{\mathbf{g}} \\
\mathbf{g}^{u}
\end{array}\right\} d \Omega .
\end{aligned}
$$

Here $\mathbf{I}$ denotes the $2 \times 2$ identity matrix, $\mathbf{p}^{g}=k \tilde{\mathbf{g}}, \mathbf{p}^{u}=k \mathbf{g}^{u}=k \operatorname{grad} \tilde{u}, \mathbf{g}^{p}=k^{-1} \tilde{\mathbf{g}}$, $\mathrm{g}^{u}=\operatorname{grad} \tilde{u}$. This notational convention, introduced in $[19,20]$, is based on two rules:

1. A varied (primary) field is marked with a superposed tilde such as $\tilde{u}$ or $\tilde{\mathbf{p}}$. This allows to reserve tildeless symbols such as $u$ or $\mathbf{p}$ for generic or exact fields.

2. A derived (secondary) field is identified by writing its "parent" primary field as superscript; e.g. $\mathbf{p}^{u}=k \operatorname{grad} \tilde{u}$ is the flux associated with the varied field $\tilde{u}$.

Of course at the exact solution of (12) all p's and g's coalesce, but the distinction is crucial in variational-based approximation methods.

Note the pleasing appearance of the last term in (18): the notation groups fluxes on the left and gradients on the right. Flux times gradient is internal energy, so the kernel matrix simply weights, through the $j$ coefficients, the nine possible combinations $\tilde{\mathbf{p}}^{T} \mathbf{g}^{p}, \tilde{\mathbf{p}}^{T} \tilde{\mathbf{g}}, \ldots$ etc. (It is possible to further streamline (18) into $U=\frac{1}{2} \int_{\Omega} \mathbf{z}^{T} \mathbf{W z} d \Omega$, as in the last of (8), but this is too compact for most developments.) These notational conventions are especially helpful in the more complicated application problems presented in Sections 5-7.

The first variation of $U$ may be compactly expressed as

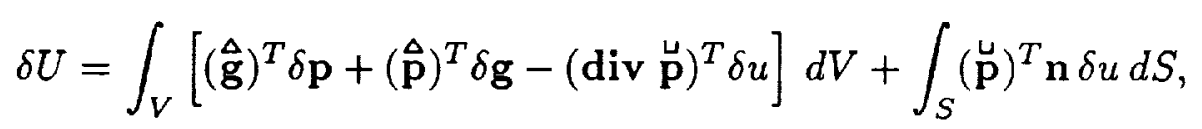

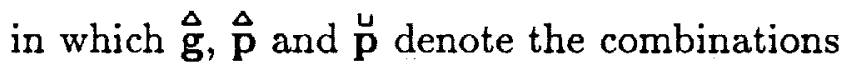

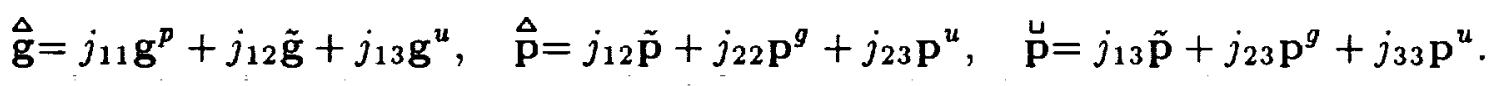

On linking $\delta U$ with the variation of the parameter-free external-energy term $P$ given in (14), we obtain the Euler-Lagrange equations in $\Omega$

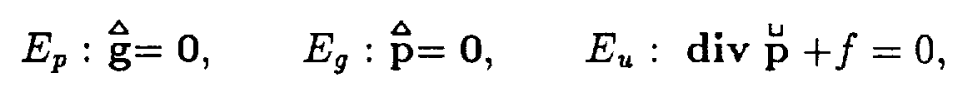

while the Neumann boundary condition on $\Gamma_{q}$ is $\stackrel{u}{q}=\left(\stackrel{\mathbf{p}}{)^{T}} \mathbf{n}=\widehat{q}\right.$. Consistency with the three field equations (16) and the boundary condition $q=\hat{q}$ leads to constraint conditions on the $j$ coefficients. These can be expeditiously obtained by noting that at the exact solution of the Poisson's problem, $\mathbf{p}=\tilde{\mathbf{p}}=\mathbf{p}^{g}=\mathbf{p}^{u}$ and $\mathbf{g}=\tilde{\mathbf{g}}=\mathbf{g}^{g}=\mathbf{g}^{u}$. Consequently

$$
j_{11}+j_{12}+j_{13}=0, \quad j_{11}+j_{12}+j_{13}=0, \quad j_{11}+j_{12}+j_{13}=1 \text {. }
$$


It follows that the functional (18) combined with $P$ yields a PVP, which is in fact a threeparameter family. If we chose the diagonal entries $j_{11}, j_{22}$ and $j_{33}$ as the free parameters, the constraints (22) are met if the functional generating matrix takes the form

$$
\mathbf{J}=\left[\begin{array}{ccc}
j_{11} & \frac{1}{2}\left(-j_{11}-j_{22}+j_{33}-1\right) & \frac{1}{2}\left(-j_{11}+j_{22}-j_{33}+1\right) \\
& j_{22} & \frac{1}{2}\left(j_{11}-j_{22}-j_{33}+1\right) \\
s y m m & & j_{33}
\end{array}\right]=\left[\begin{array}{ccc}
j_{11} & -s_{3} & -s_{2} \\
-s_{3} & j_{22} & -s_{1} \\
-s_{2} & -s_{1} & j_{33}
\end{array}\right] .
$$

Here the negatives of the three off-diagonal elements of $\mathbf{J}$ are abbreviated to $s_{1}, s_{2}$ and $s_{3}$ for use below.

The choice $j_{33}=1$, others zero, yields the well known single-field functional (16). Other choices for $\mathbf{J}$ are discussed in conjunction with the classical elasticity problem in Section 5 , which has a similar parametric structure.

Using the decomposition of $\mathrm{J}$ as sum of rank-one matrices

$$
\mathbf{J}=\left[\begin{array}{lll}
0 & 0 & 0 \\
0 & 0 & 0 \\
0 & 0 & 1
\end{array}\right]+s_{1}\left[\begin{array}{rrr}
0 & 0 & 0 \\
0 & 1 & -1 \\
0 & -1 & 1
\end{array}\right]+s_{2}\left[\begin{array}{rrr}
1 & 0 & -1 \\
0 & 0 & 0 \\
-1 & 0 & 1
\end{array}\right]+s_{3}\left[\begin{array}{rrr}
1 & -1 & 0 \\
-1 & 1 & 0 \\
0 & 0 & 0
\end{array}\right]
$$

one can rewrite the three Euler-Lagrange equations (21) in a form that illuminates the weighted-residual connection to the field equations (16):

$$
\begin{aligned}
& E_{p}: s_{2}\left(\mathbf{g}^{p}-\mathbf{g}^{u}\right)+s_{3}\left(\mathbf{g}^{p}-\tilde{\mathbf{g}}\right)=0, \\
& E_{g}: s_{3}\left(\mathbf{p}^{g}-\tilde{\mathbf{p}}\right)+s_{1}\left(\mathbf{p}^{g}-\mathbf{p}^{u}\right)=0, \\
& E_{u}: \operatorname{div}\left[\mathbf{p}^{u}+s_{1}\left(\mathbf{p}^{u}-\mathbf{p}^{g}\right)+s_{2}\left(\mathbf{p}^{u}-\tilde{\mathbf{p}}\right)\right]+f=0 .
\end{aligned}
$$

What happens if, say, $s_{2}=s_{3}=0$ ? Then $E_{p}$ becomes an identity and $\tilde{\mathbf{g}}$ drops out as an independently varied field [observe that $j_{11}=0$ because of (22) and consequently the first row and column of $\mathbf{J}$ vanish.] Similarly if $s_{1}=s_{3}=0, E_{g}$ becomes an identity and $\tilde{\mathbf{p}}$ drops out as varied field. The case $s_{1}=s_{2}=0$ reduces $E_{u}$ to $\operatorname{div} \mathrm{p}^{u}+f=0$ but three-field principles are still possible because one may select $j_{11}=j_{22}=-j_{12}=\rho$, where $\rho$ is arbitrary; setting $\rho=0$ gives back the simplified functional (14).

The form (25) show that the $s$ 's, or their reciprocals, can be interpreted as weights on the field equations. No such flexibility is available with single-field functionals because the parameters factor out at the first variation level. This is the key reason behind the importance of multifield PVPs.

Is this PVP invariant?. At an extremal the p's and g's coalesce. The internal energy reduces to $\int_{\Omega} \mathbf{P}^{T} \mathbf{g} d V$ multiplied by $j_{11}+j_{12}+\ldots+j_{33}$, which is one because of (22). Thus we have an IPVP. The same property holds for all PVPs presented in Sections 5-8, and is crucial in the application to finite element error-estimation discussed in Section 10.

Does (18) embed all possible functionals for the internal energy of the Poisson's equation? No! In this formulation $u$ must be a primary field in $\Pi$, although $\tilde{\mathbf{p}}$ and/or $\tilde{\mathbf{g}}$ may drop 


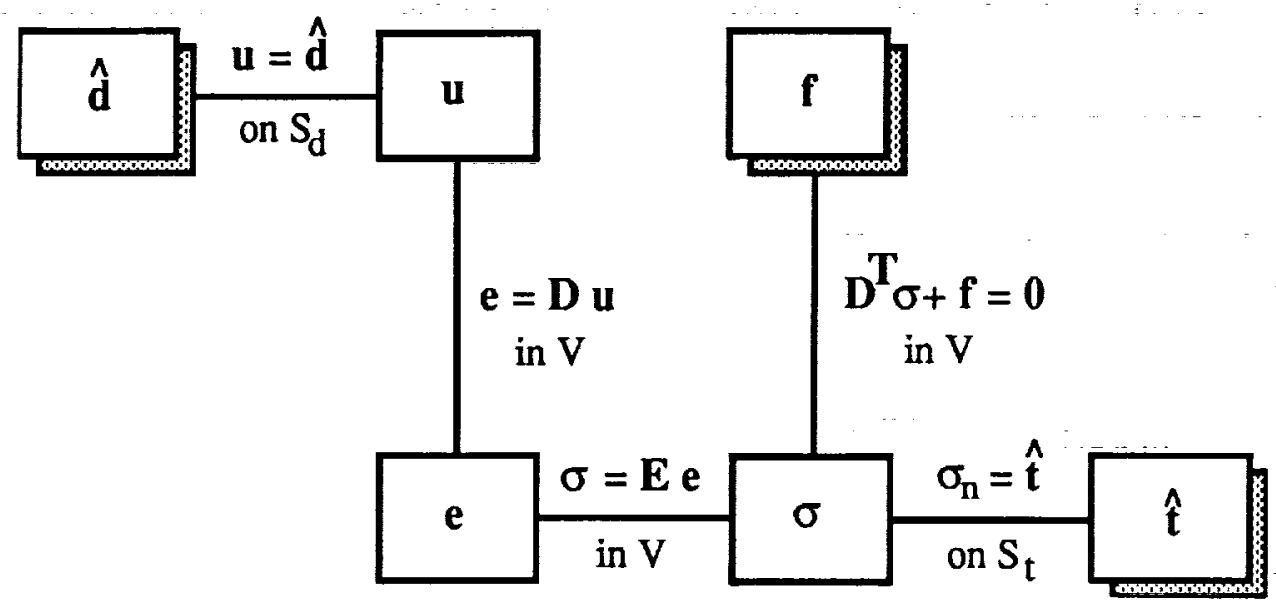

Fig. 3. Graphical representation of the Strong Form (SF) of the primal (displacement-based) formulation of classical elasticity. Refer to Figure 2 for display conventions.

out for certain choices of $\mathbf{J}$ as discussed above. To make $u$ disappear, the last row (and column) of $\mathbf{J}$ must have all zero entries, which contradicts the last of (22). Consequently functionals with internal energy of the form $U(\mathbf{p}, \mathbf{g}), U(\mathbf{p})$ or $U(\mathbf{g})$ escape this framework. This point is further elaborated in Section 5.5.

\section{CLASSICAL ELASTICITY}

"Classical elasticity" is short for the more precise "compressible linear hyperelastotatics." This is the application area in which the multifield PVPs discussed here originated in response to needs from finite element technology. As a result, it is still the best developed one in terms of FEM applications.

The ensuing discussion emphasizes three-dimensional elasticity. Specialization to structural models such as beams, plates and shells, as well as the derivation of canonical functionals for these cases, is covered from a modern viewpoint by several authors, e.g. Reddy $[53,54]$ and Hartmann [31].

\subsection{Governing Equations}

Consider an body of volume $V$ referred to a rectangular Cartesian coordinate system $x_{i}$, $i=1,2,3$. The body is bounded by surface $S$ of external unit normal $\mathbf{n} \equiv n_{i}$. The surface is decomposed into $S: S_{d} \cup S_{t}$. Displacements $\widehat{\mathrm{d}} \equiv \hat{d}_{i}$ are prescribed on $S_{d}$ whereas surface tractions $\widehat{\mathbf{t}}=\hat{t}_{i}$ are prescribed on $S_{t}$. Body forces $\mathbf{f} \equiv f_{i}$ are prescribed in volume $V$.

The three unknown internal fields are: displacements $\mathbf{u} \equiv u_{i}$, strains $\mathrm{e} \equiv e_{i j}$ and stresses $\sigma \equiv \sigma_{i j}$. The stress traction vector on $S$ is $\sigma_{n} \equiv \sigma_{n i}=\sigma_{j i} n_{j}$ (summation convention implied). To facilitate the construction of elasticity functionals in matrix form, stresses 
and strains are arranged in the usual 6-component vector forms

$$
\begin{aligned}
\boldsymbol{\sigma}^{T} & =\left[\begin{array}{llllll}
\sigma_{11} & \sigma_{22} & \sigma_{33} & \sigma_{12} & \sigma_{23} & \sigma_{31}
\end{array}\right] \\
\mathbf{e}^{T} & =\left[\begin{array}{llllll}
e_{11} & e_{22} & e_{33} & 2 e_{12} & 2 e_{23} & 2 e_{31}
\end{array}\right]
\end{aligned}
$$

These fields are connected by the kinematic, constitutive and balance equations

$$
\mathrm{e}=\mathrm{Du}, \quad \boldsymbol{\sigma}=\mathrm{Ee}, \quad \mathrm{D}^{T} \boldsymbol{\sigma}+\mathbf{f}=\mathbf{0}
$$

where

$$
\mathbf{D}=\left[\begin{array}{ccc}
\partial / \partial x_{1} & 0 & 0 \\
0 & \partial \partial x_{2} & 0 \\
0 & 0 & \partial / \partial x_{3} \\
\partial / \partial x_{2} & \partial / \partial x_{1} & 0 \\
0 & \partial / \partial x_{3} & \partial / \partial x_{2} \\
\partial / \partial x_{3} & 0 & \partial / \partial x_{1}
\end{array}\right]
$$

is the $6 \times 3$ symmetric-gradient operator, its transpose $\mathbf{D}^{T}$ the $3 \times 6$ tensor-divergence operator, and $\mathrm{E}$ is the $6 \times 6$ stress-strain matrix of elastic moduli arranged in the usual manner. The boundary conditions are

$$
\mathbf{u}=\widehat{\mathbf{d}} \quad \text { on } S_{d}, \quad \sigma_{n}=\widehat{\mathbf{t}} \quad \text { on } S_{t} .
$$

The field equations (27) and boundary conditions (29) make up the Strong Form (SF) of the classical-elasticity problem. The SF is graphically represented in Figure 3 using again a modified Tonti diagram of the primal (displacement-based) formulation.

\subsection{Weak and Variational Forms}

From the Strong Form one can proceed to several Weak Forms (WF) by selectively "weakening" strong connections. A variation-homogenization and integration process then yields a Variational Form (VF) as was sketched in Figure 1. Two specific functionals of classical elasticity are worked out as examples before proceeding to the general parametrization.

Figure 4 depicts the Weak Form (WF) of the Potential Energy (PE) functional for classical elastostatics, in which the displacement field $\mathbf{u}$ is the only primary field. The WF diagram is adapted from the Strong-Form Tonti diagrams of Figures 2-3, with some additional graphic conventions explained in Figure 4. Furthermore the derived field notation introduced in Section 4.3 appears again. For example, $\mathbf{e}^{u}=\mathrm{Du}$ is identified by the "tracer superscript" $u$ that remind us that these are displacement-derived strains.

Two strong connections: the balance equations $\mathbf{D}^{T} \boldsymbol{\sigma}+\mathbf{f}=\mathbf{0}$ in $V$ and the flux boundary condition: $\sigma_{n}^{u}=\widehat{t}$ on $S_{t}$, have been weakened by making them satisfied only in an average sense:

$$
\int_{V}\left(\mathbf{D}^{T} \boldsymbol{\sigma}^{u}+\mathbf{f}\right) \mathbf{w} d V+\int_{S_{\mathbf{t}}}\left(\boldsymbol{\sigma}_{n}^{u}-\hat{\mathbf{t}}\right) \mathbf{w} d S=0
$$




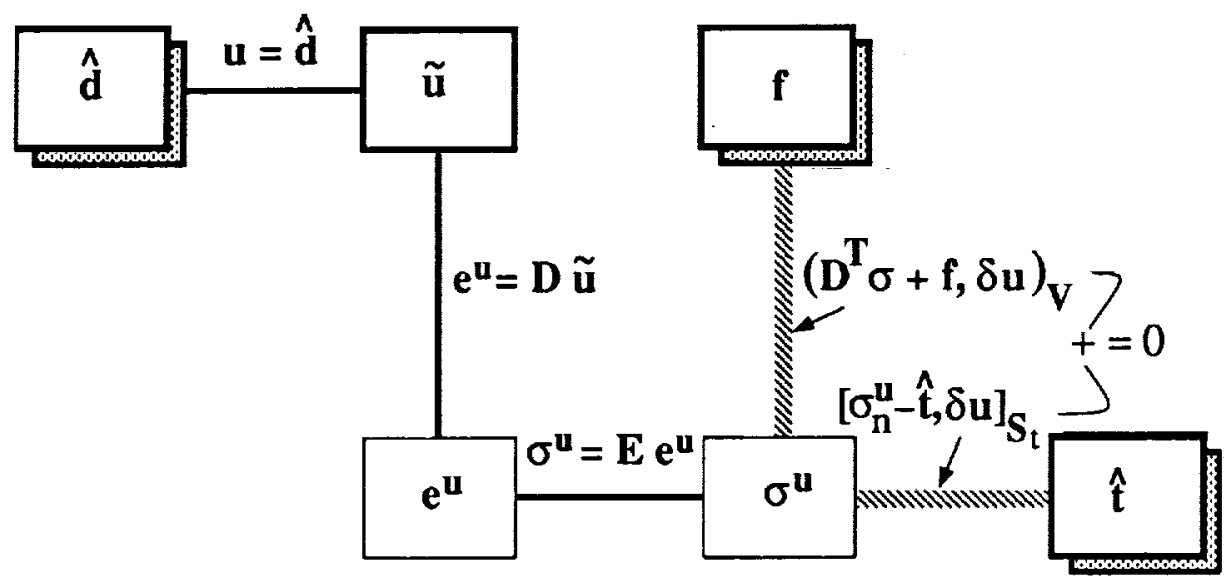

Varied (primary) field
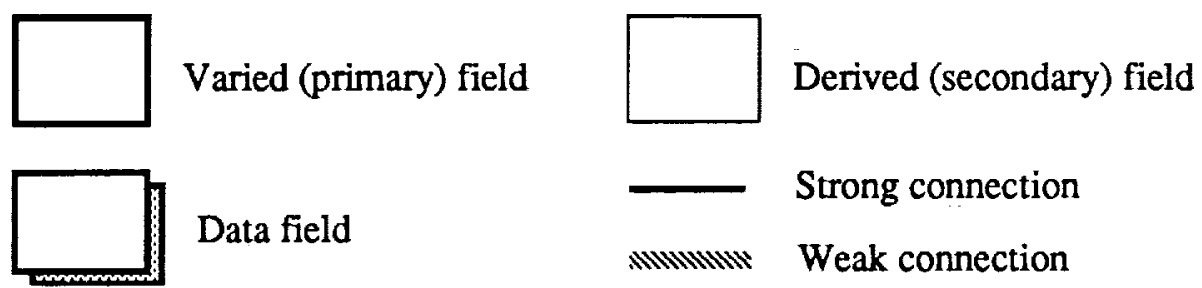

Data field

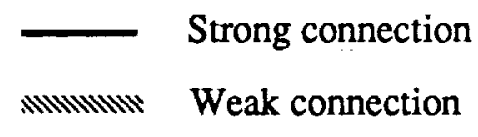

Fig. 4. The Weak Form (WF) of the Potential Energy principle of classical elasticity. Also known as the Principle of Virtual Work. The function inner-product abbreviations $(\mathbf{a}, \mathbf{b})_{V} \stackrel{\text { def }}{=} \int_{V} \mathbf{a}^{T} \mathbf{b} d V$ and $[\mathbf{a}, \mathbf{b}]_{s} \stackrel{\text { def }}{=} \int_{S} \mathbf{a}^{T} \mathbf{b} d S$ are used for labeling the weak connections because $\int$ is not available for graphics.

where $w$ are the "virtual displacements" in the parlance of mechanics. To pass to the VF, set $\mathbf{w}=\delta \mathbf{u}$, homogenize variations and integrate obtaining the Potential Energy functional

$$
\Pi_{P E}(\tilde{\mathbf{u}})=\frac{1}{2} \int_{V}\left(\boldsymbol{\sigma}^{u}\right)^{T} \mathbf{e}^{u} d V-\int_{V} \mathbf{f}^{T} \tilde{\mathbf{u}} d V-\int_{S_{\mathbf{t}}} \hat{\mathbf{t}}^{T} \tilde{\mathbf{u}} d S
$$

As second example, consider the derivation of the Hellinger-Reissner (HR) stressdisplacement functional. Figure 5 depicts the appropriate Weak Form (WF). The varied fields are displacements $\tilde{\mathbf{u}}$ and stresses $\tilde{\sigma}$. Three weak links have been introduced:

$$
\int_{V}\left(\mathbf{e}^{u}-\mathbf{e}^{\sigma}\right) \delta \boldsymbol{\sigma} d V+\int_{V}\left(\mathbf{D}^{T} \boldsymbol{\sigma}+\mathbf{f}\right) \delta \mathbf{u} d V+\int_{S_{\mathfrak{\imath}}}\left(\tilde{\boldsymbol{\sigma}}_{n}-\widehat{\mathbf{t}}\right) \delta \mathbf{u} d S=0
$$

where $\tilde{\sigma}_{n} \equiv \tilde{\sigma}_{i j} n_{j}$. From this one can pass to the Hellinger-Reissner functional

$$
\Pi_{H R}(\tilde{\mathbf{u}}, \tilde{\boldsymbol{\sigma}})=\int_{V} \tilde{\boldsymbol{\sigma}}^{T}\left(\mathbf{e}^{u}-\frac{1}{2} \mathbf{e}^{\sigma}\right) d V-\int_{V} \mathbf{f}^{T} \tilde{\mathbf{u}} d V-\int_{S_{\mathbf{t}}} \widehat{\mathbf{t}}^{T} \tilde{\mathbf{u}} d S .
$$

There are variants of both the PE and HR functionals in which the displacement boundary condition $\mathbf{u}=\widehat{\mathbf{d}}$ is only weakly enforced. This results in the appearance of an additional $S_{d}$ boundary term displayed in (35) below. 


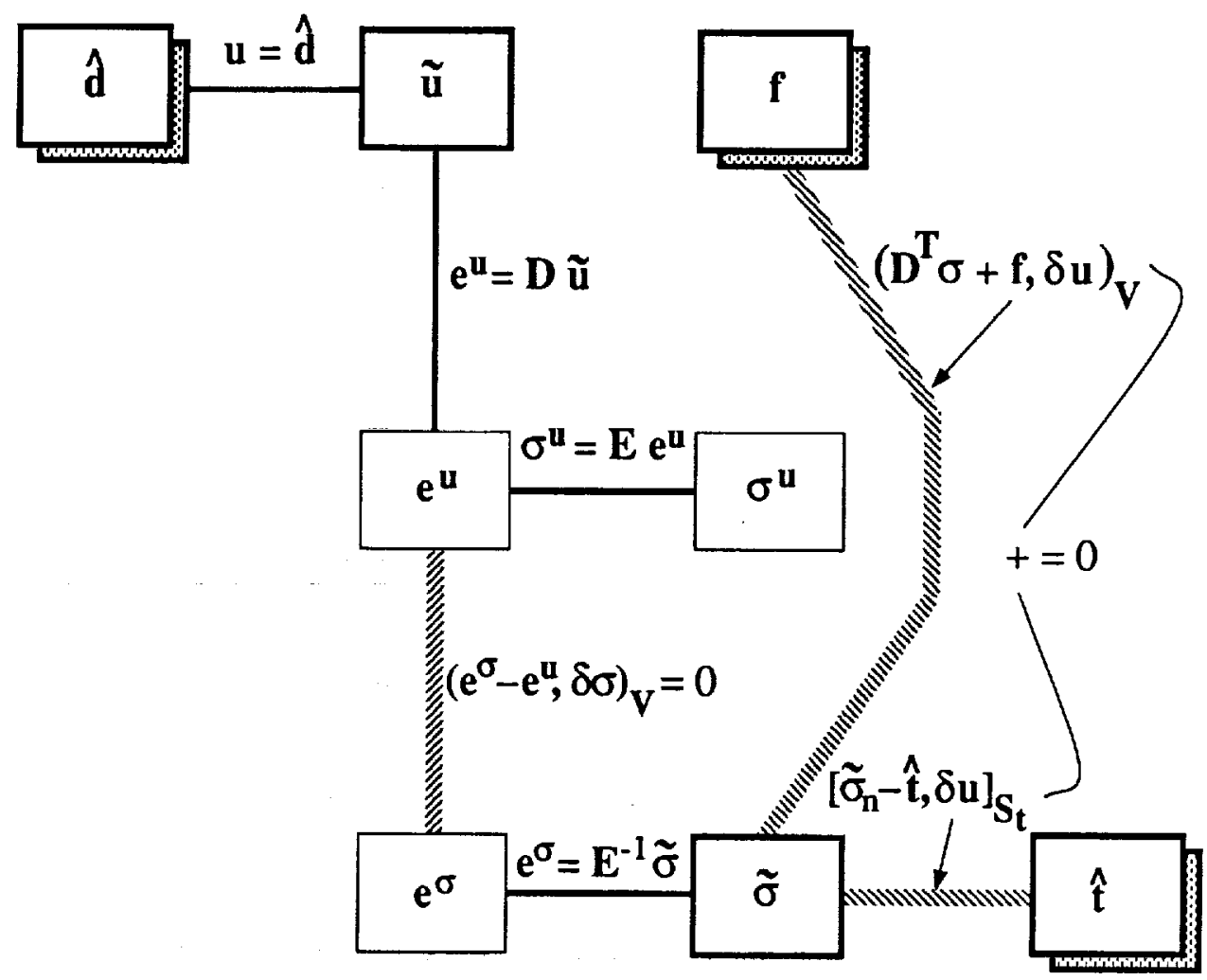

Fig. 5. The Weak Form (WF) of the Hellinger-Reissner principle of classical elasticity. (For notation explanation refer to Figure 4.)

\subsection{Canonical Functionals and Hybridization}

The PE and HR functionals are instances of the so-called canonical functionals. According to Oden and Reddy $[46,47]$ there are seven canonical functionals for the primal formulation of linear selfadjoint boundary value problems that fit the SF scheme of Figures 2 and 3. For classical linear elastostatics these are listed in Table 1, along with the names of the best known ones. (Oden and Reddy list eight functionals but one is a trivial combination of $\mathrm{PE}$ and HR.) This number expands to 14 if one includes the dual formulation of elasticity based on potentials, but such generalization - which is discussed in detail by Oden and Reddy [47] — will not be considered here.

Are there more? The number increases if one allows hybrid functionals into consideration. All functionals of classical elasticity can be expressed in the form analogous to (14):

$$
\Pi=U-P,
$$

where $U$ is the volume term that represents stored strain energy and the potential $P$ includes body-force and boundary contributions. The conventional form of $P$ is:

$$
P^{c}(\tilde{\mathbf{u}}, \tilde{\boldsymbol{\sigma}}, \tilde{\mathbf{e}})=\int_{V} \mathbf{f}^{T} \tilde{\mathbf{u}} d V+\int_{S_{d}}\left(\breve{\boldsymbol{\sigma}}_{n}\right)^{T}(\tilde{\mathbf{u}}-\widehat{\mathbf{d}}) d S+\int_{S_{\mathbf{t}}} \hat{\mathbf{t}}^{T} \tilde{\mathbf{u}} d S
$$


Table 1. The Canonical Functionals of Classical Linear Elastostatics

(after Oden and Reddy [47])

\begin{tabular}{|ccl|}
\hline Varied fields & Acronym & Functional name \\
\hline$\tilde{\mathbf{u}}$ & PE & Potential energy \\
$\tilde{\boldsymbol{\sigma}}$ & $\mathrm{CE}$ & Complementary energy \\
$\tilde{\mathbf{e}}$ & & No name \\
$\tilde{\mathbf{u}}, \tilde{\boldsymbol{\sigma}}$ & HR & Hellinger-Reissner \\
$\tilde{\mathbf{u}} \tilde{\mathbf{e}}$ & SDR & Strain-displacement Reissner \\
$\tilde{\boldsymbol{\sigma}}, \tilde{\mathbf{e}}$ & & No name \\
$\tilde{\mathbf{u}}, \tilde{\boldsymbol{\sigma}}, \tilde{\mathbf{e}}$ & HW & Hu-Washizu \\
\hline
\end{tabular}

where $\breve{\sigma}_{n}$ is generally a weighted combination of $\sigma^{u}, \sigma^{e}$ and $\sigma$ as shown in Eq. (43) of Section 5.4. The $S_{d}$ term drops if the displacement boundary condition $\mathbf{u}=\widehat{\mathbf{d}}$ is strong, as in (31) and (33); in such case $P^{c}$ depends only on $\tilde{\mathbf{u}}$.

Two more forms appear when one considers hybrid functionals for a finite element discretization of $V$. (Such functionals can also be constructed for bodies with internal physical interfaces.) In the following $S_{i}$ is the counted-twice union of element interfaces not on $S$, while $\tilde{\mathbf{d}}$ and $\tilde{\mathbf{t}}$ are boundary-displacement and boundary-traction fields, respectively, that are independently varied on $S_{i}$ :

$$
\begin{gathered}
P^{d}(\tilde{\mathbf{u}}, \tilde{\boldsymbol{\sigma}}, \tilde{\mathbf{e}}, \tilde{\mathbf{d}})=\int_{V} \mathbf{f}^{T} \tilde{\mathbf{u}} d V+\int_{S_{d}}\left(\breve{\boldsymbol{\sigma}}_{n}\right)^{T}(\tilde{\mathbf{u}}-\widehat{\mathbf{d}}) d S+\int_{S_{\mathbf{t}}} \hat{\mathbf{t}}^{T} \tilde{\mathbf{u}} d S+\int_{S_{i}} \boldsymbol{\sigma}_{n}^{T}(\tilde{\mathbf{u}}-\tilde{\mathbf{d}}) d S . \\
P^{t}(\tilde{\mathbf{u}}, \tilde{\mathbf{t}})=\int_{V} \mathbf{f}^{T} \tilde{\mathbf{u}} d V+\int_{S_{d}} \tilde{\mathbf{t}}^{T}(\tilde{\mathbf{u}}-\widehat{\mathbf{d}}) d S+\int_{S_{\mathbf{t}}} \hat{\mathbf{t}}^{T} \tilde{\mathbf{u}} d S+\int_{S_{\mathbf{i}}} \tilde{\mathbf{t}}^{T} \tilde{\mathbf{u}} d S .
\end{gathered}
$$

Use of either of these potentials allows $\tilde{\mathbf{u}}$ to be discontinuous across internal interfaces. In (36) fields $\tilde{\mathbf{d}}$ and $\tilde{\mathbf{u}}$ are weakly connected on $S_{\boldsymbol{i}}$, whereas in (37) fields $\tilde{\mathbf{t}}$ and $\breve{\sigma}_{n}$ are weakly connected on $S_{i}$. With these additional choices one can have, for example, three variants of the Hellinger-Reissner functional: $\Pi_{H R}^{c}=U_{H R}-P^{c}, \Pi_{H R}^{d}=U_{H R}-P^{d}$, and $\Pi_{H R d}^{t}=U_{H R}-P^{t}$. The last two were called $d$-generalized and $t$-generalized functionals, respectively, in $[19,20]$. The hybrid functionals of Pian and Tong $[51,52,63]$ can be precipitated as special cases of these two forms.

When these hybrid variants are accounted for, the number of primal canonical functionals roughly triples (it is not exactly $7 \times 3=21$ because certain hybridizations are incompatible with some functionals of Table 1). But in fact all of them are mere "points" in the space of parametrized functionals constructed below. Therefore the correct answer to the previous question is that there is an infinite number of functionals from which classical elasticity can be derived. 


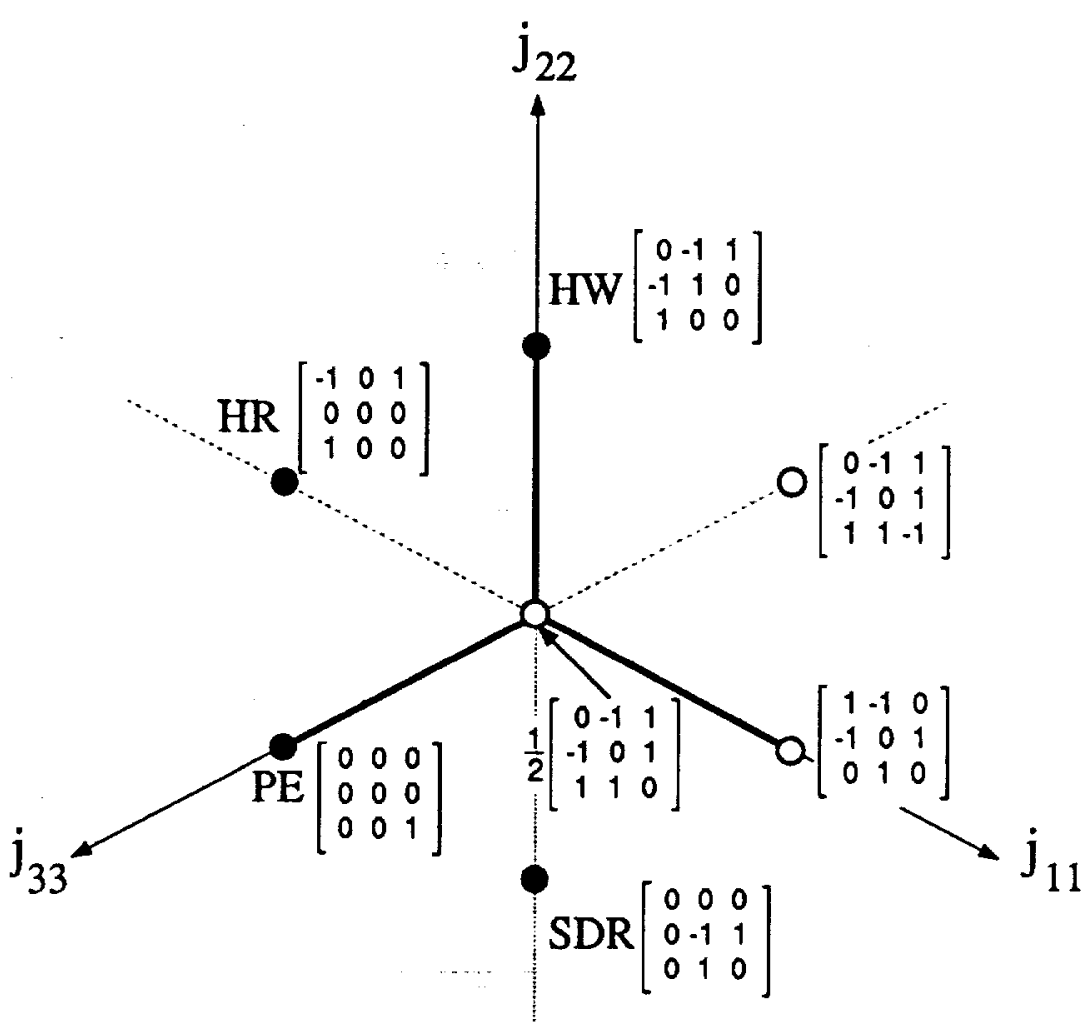

Fig. 6. Representation of the three-parameter PVP for classical elasticity in $\left(j_{11}, j_{22}, j_{33}\right)$ space. Generating matrices for interesting functionals are shown near the "points."

\subsection{Heuristic Parametrization}

The strain energy portion $U$ of $\Pi=U-P$ can be written down as follows for the PE, HR and HW canonical functionals:

$$
\begin{gathered}
U_{P E}(\tilde{\mathbf{u}})=\frac{1}{2} \int_{V}\left\{\begin{array}{c}
\tilde{\boldsymbol{\sigma}} \\
\boldsymbol{\sigma}^{e} \\
\boldsymbol{\sigma}^{u}
\end{array}\right\}^{T}\left[\begin{array}{ccc}
\mathbf{0} & \mathbf{0} & 0 \\
\mathbf{0} & \mathbf{0} & \mathbf{0} \\
\mathbf{0} & \mathbf{0} & \mathbf{I}
\end{array}\right]\left\{\begin{array}{c}
\mathbf{e}^{\sigma} \\
\tilde{\mathbf{e}} \\
\mathbf{e}^{u}
\end{array}\right\} d V, \\
U_{H R}(\tilde{\mathbf{u}}, \tilde{\boldsymbol{\sigma}})=\frac{1}{2} \int_{V}\left\{\begin{array}{c}
\tilde{\boldsymbol{\sigma}} \\
\boldsymbol{\sigma}^{e} \\
\boldsymbol{\sigma}^{u}
\end{array}\right\}^{T}\left[\begin{array}{ccc}
-\mathbf{I} & \mathbf{0} & \mathbf{I} \\
\mathbf{0} & \mathbf{0} & 0 \\
\mathbf{I} & \mathbf{0} & \mathbf{0}
\end{array}\right]\left\{\begin{array}{c}
\mathbf{e}^{\sigma} \\
\tilde{\mathbf{e}} \\
\mathbf{e}^{u}
\end{array}\right\} d V, \\
U_{H W}(\tilde{\mathbf{u}}, \tilde{\boldsymbol{\sigma}}, \tilde{\mathbf{e}})=\frac{1}{2} \int_{V}\left\{\begin{array}{c}
\tilde{\boldsymbol{\sigma}} \\
\boldsymbol{\sigma}^{e} \\
\boldsymbol{\sigma}^{u}
\end{array}\right\}^{T}\left[\begin{array}{ccc}
\mathbf{0} & -\mathbf{I} & \mathbf{I} \\
-\mathbf{I} & \mathbf{I} & \mathbf{0} \\
\mathbf{I} & \mathbf{0} & \mathbf{0}
\end{array}\right]\left\{\begin{array}{c}
\mathbf{e}^{\sigma} \\
\tilde{\mathbf{e}} \\
\mathbf{e}^{u}
\end{array}\right\} d V,
\end{gathered}
$$

where $I$ is the $6 \times 6$ identity matrix. This pattern suggests trying the parametrized form

$$
U(\mathbf{u}, \boldsymbol{\sigma}, \mathbf{e})=\frac{1}{2} \int_{V}\left\{\begin{array}{c}
\boldsymbol{\sigma} \\
\boldsymbol{\sigma}^{e} \\
\boldsymbol{\sigma}^{u}
\end{array}\right\}^{T}\left[\begin{array}{lll}
j_{11} \mathbf{I} & j_{12} \mathbf{I} & j_{13} \mathbf{I} \\
j_{12} \mathbf{I} & j_{22} \mathbf{I} & j_{23} \mathbf{I} \\
j_{13} \mathbf{I} & j_{23} \mathbf{I} & j_{33} \mathbf{I}
\end{array}\right]\left\{\begin{array}{c}
\mathbf{e}^{\sigma} \\
\mathbf{e} \\
\mathbf{e}^{u}
\end{array}\right\} d V
$$


This is formally similar to (18) except that the $\sigma$ and e vectors have 6 components, and the generalized field vectors 18 components.

The first variation of $U$ can be compactly written

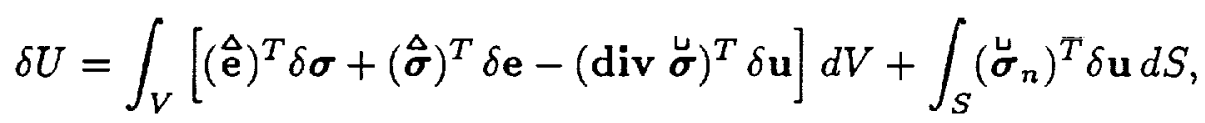

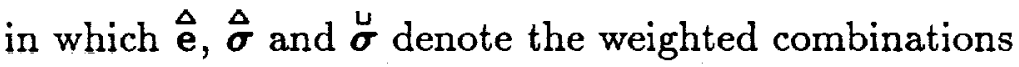

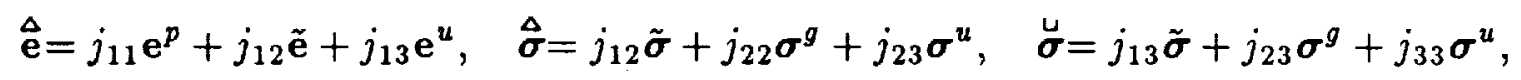

Combining $\delta U$ with $\delta P$ yields $\delta \Pi$. For example, if $P$ is taken as $P^{c}$ of (35)

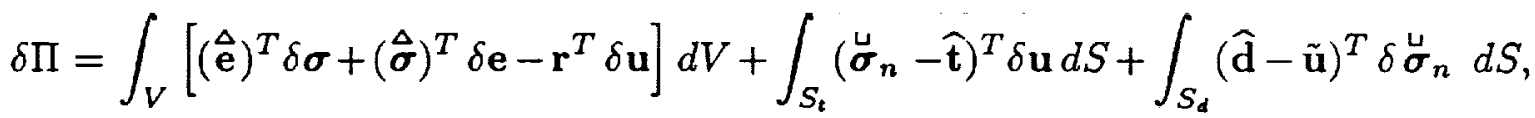

where $\mathbf{r}=\operatorname{div} \stackrel{\boldsymbol{\sigma}}{+} \mathbf{f}$ are internal equilibrium violations. Consistency arguments again show that the coefficients must satisfy the constraints (22) reproduced here for convenience:

$$
j_{11}+j_{12}+j_{13}=0, \quad j_{12}+j_{22}+j_{23}=0, \quad j_{13}+j_{23}+j_{33}=1
$$

This leaves $6-3=3$ free parameters. As usual the $3 \times 3$ matrix $\mathbf{J}$ of entries $j_{k \ell}$ is called the functional generating matrix. The special settings

$$
\mathbf{J}=\left[\begin{array}{lll}
0 & 0 & 0 \\
0 & 0 & 0 \\
0 & 0 & 1
\end{array}\right], \quad \mathbf{J}=\left[\begin{array}{ccc}
-1 & 0 & 1 \\
0 & 0 & 0 \\
-1 & 0 & 0
\end{array}\right], \quad \mathbf{J}=\left[\begin{array}{ccc}
0 & -1 & 1 \\
-1 & 1 & 0 \\
1 & 0 & 0
\end{array}\right], \quad \mathbf{J}=\left[\begin{array}{ccc}
0 & 0 & 0 \\
0 & -1 & 1 \\
0 & 1 & 0
\end{array}\right],
$$

yield the PE, HR, HW and SDR canonical functionals, respectively. Other interesting but anonymous functionals in $j_{11}, j_{22}, j_{33}$ space are marked with a $\circ$ in Figure 6 . A glance at this figure derails the lofty status accorded the $\mathrm{Hu}$-Washizu (HW) functional in many textbooks as the most general three-field functional of elasticity.

The weighted field-equation representation analogous to (25) is

$$
\begin{aligned}
& E_{e}: s_{2}\left(\mathbf{e}^{\sigma}-\mathbf{e}^{u}\right)+s_{3}\left(\mathbf{e}^{\sigma}-\tilde{\mathbf{e}}\right)=0 \\
& E_{\sigma}: s_{3}\left(\boldsymbol{\sigma}^{e}-\tilde{\boldsymbol{\sigma}}\right)+s_{1}\left(\boldsymbol{\sigma}^{e}-\boldsymbol{\sigma}^{u}\right)=\mathbf{0} \\
& E_{u}: \operatorname{div}\left[\boldsymbol{\sigma}^{u}+s_{1}\left(\boldsymbol{\sigma}^{u}-\boldsymbol{\sigma}^{e}\right)+s_{2}\left(\boldsymbol{\sigma}^{u}-\tilde{\boldsymbol{\sigma}}\right)\right]+\mathbf{f}=0,
\end{aligned}
$$

where scaling factors $s_{1}, s_{2}$ and $s_{3}$ are defined in (23). The general Weak Form for arbitrary parameters is depicted in Figure 7. As can be observed this has become more complex, requiring some study to sort out. The "strength" of the weak connections can be measured by the value of the scaling factors as discussed in Section 4.3. If factor pairs vanish "box merging" occurs as explained therein. 

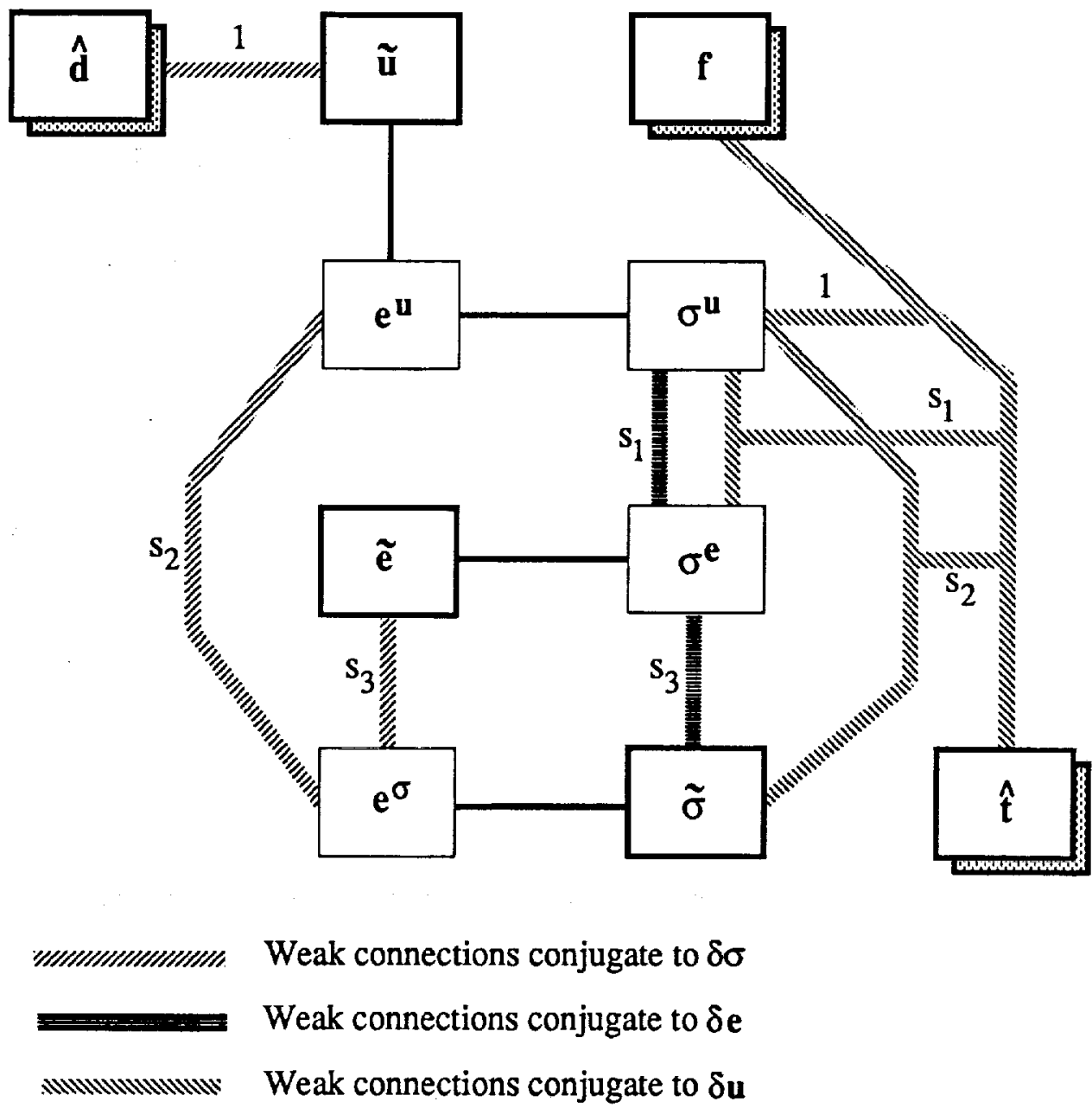

Fig. 7. Weak Form for the general parametrization (40). Numbers annotated near weak connections are the scaling factors in Eqs. (46).

\subsection{Functionals Without Independent Displacements}

There is a subset of elasticity functionals without independently varied displacements; for example the well known principle of Complementary Energy (CE). In Felippa and Militello [22] it is shown that all functionals with independently varied stresses can be embedded in a one-parameter form that includes $\mathrm{CE}$ as a special case. The only functional left out of these three-parameter and one-parameter framework is the unnamed canonical functional whose only independent varied field is strains. Summarizing: all canonical primal functionals of linear elasticity can be covered with a 3-parameter family $\Pi(\tilde{\mathbf{u}}, \tilde{\boldsymbol{\sigma}}, \tilde{\mathbf{e}})$, a 1-parameter family $\Pi(\tilde{\boldsymbol{\sigma}}, \tilde{\mathbf{e}})$, plus a 0 -parameter family $\Pi(\tilde{\mathbf{e}})$. This statement can be extended to encompass hybrid variants.

\subsection{Historical Note}

The three-parameter form (41) was not the first one constructed. A one-parameter subset that connects points PE and HR of Figure 6 was discovered in 1987 and published in 


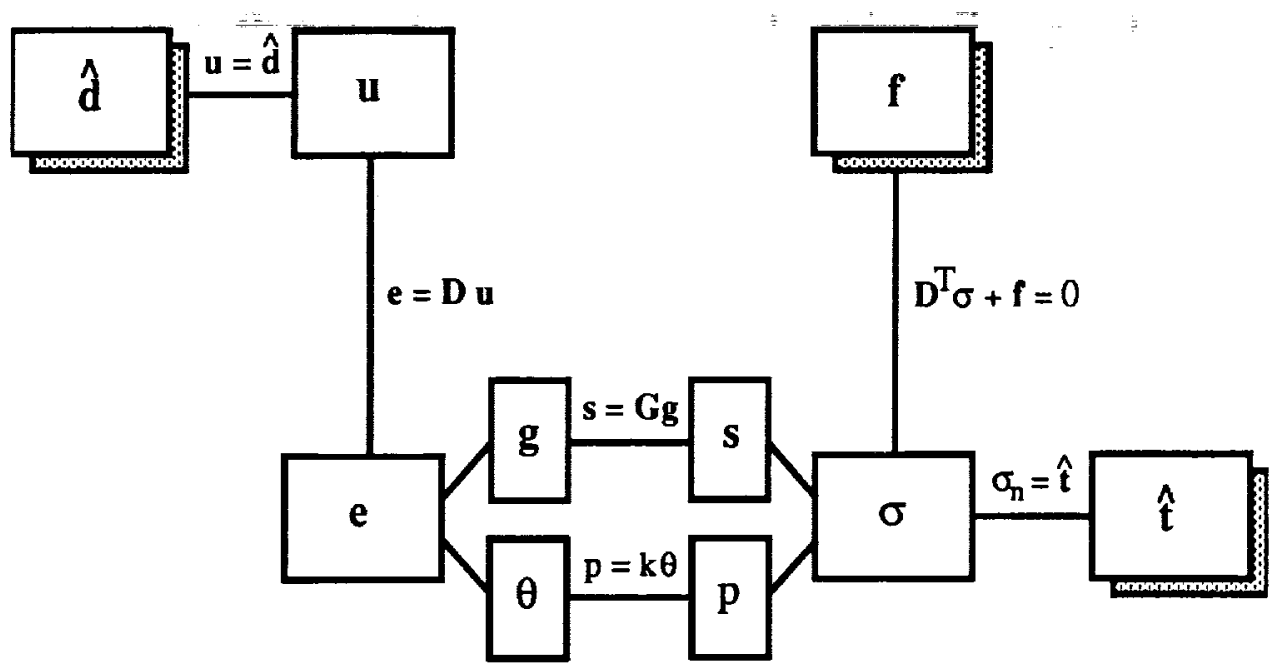

Fig. 8. Generalization of the Strong Form of classical linear elastostatics (diagramed in Figure 3) to encompass material incompressibility.

$[19,20]$. This was the byproduct of an effort to variationally justify the Free Formulation (FF) of finite elements [12,13]. The effort was actually motivated by criticism of a followup paper [18] by a reviewer who justly observed that the excellent performance of the FF-based plate-bending element presented therein had computational but no theoretical basis.

Subsequent developments were serendipitous. Another one-parameter form, joining "points" HR and HW of Figure 6, had been constructed $[40,41]$ to justify the Assumed Natural Strain method $[7,32,50,58]$. This work evolved later into the Assumed Natural Deviatoric Strain (ANDES) formulation $[22,43]$. Comparison of the SFF and ANDES results led to the general parametrization (41) published in $[22,23]$.

\section{ENCOMPASSING INCOMPRESSIBLE BEHAVIOR}

The functionals of linear elastostatics studied in the previous section fail if the material is incompressible. To encompass incompressibility one must begin by splitting the stress tensor into deviatoric and mean stresses, and the strain tensor into deviatoric and mean strains. In Cartesian tensor notation:

$$
\begin{aligned}
& \sigma_{i j}=s_{i j}-p \delta_{i j}, \quad p=-\frac{1}{3} \sigma_{i i}=-\frac{1}{3} \operatorname{trace} \sigma, \\
& e_{i j}=g_{i j}-\frac{1}{3} \theta \delta_{i j}, \quad \theta=-e_{i i}=-\operatorname{trace} \mathbf{e}=-\operatorname{div} \mathbf{u} .
\end{aligned}
$$

Here $p$ denotes the (actual) pressure, $\theta$ the total strain condensation, $\delta_{i j}$ is the Kronecker delta, and the summation convention applies over the range $1,2,3$. These tensor components are arranged in 6-vector form following the prescription (26) so we can rewrite the splitting (48) as

$$
\boldsymbol{\sigma}=\mathbf{s}-p \mathbf{h}, \quad \mathbf{e}=\mathbf{g}-\theta \mathbf{h}, \quad \mathbf{h}^{T}=\left[\begin{array}{llllll}
1 & 1 & 1 & 0 & 0 & 0
\end{array}\right] .
$$


The kinematic relations and balance equations are still $\mathbf{e}=\mathbf{D u}$ and $\mathbf{D}^{T} \boldsymbol{\sigma}+\mathbf{f}=\mathbf{0}$ as in (27). The boundary conditions are still (29). As far as the constitutive equations is concerned, the assumed split is

$$
\mathbf{s}=\mathbf{G g}, \quad p=k \theta,
$$

where $\mathbf{G}$ is a (generally anisotropic) deviatoric-stress-to-deviatoric-strain matrix invertible for all values of compressibility, and $k$ is thrice the bulk modulus $K$. Incompressibility occurs in the limit $k \rightarrow \infty$. Note that the model (50) assumes that the material is volumetrically isotropic although deviatoric anisotropy is permitted. The Strong Form diagram shown in Figure 8 summarizes the governing equations.

The governing functionals are again $\Pi=U-P$. The internal energy $U$ has been parametrized by Felippa [24] in the form

$$
U(\tilde{\mathbf{u}}, \tilde{\mathbf{s}}, \tilde{p}, \tilde{\mathbf{g}}, \tilde{\theta})=\frac{1}{2} \int_{V}\left\{\begin{array}{c}
\mathbf{s} \\
\mathbf{s}^{g} \\
\mathbf{s}^{u} \\
\tilde{p} \\
p^{\theta} \\
p^{u}
\end{array}\right\} \quad\left[\begin{array}{cccccc}
j_{11} I & j_{12} I & j_{13} I & j_{14} \mathbf{h} & j_{15} \mathbf{h} & j_{16} \mathbf{h} \\
j_{12} I & j_{22} I & j_{23} \mathbf{I} & j_{24} \mathbf{h} & j_{25} \mathbf{h} & j_{26} \mathbf{h} \\
j_{13} I & j_{23} \mathbf{I} & j_{33} \mathbf{I} & j_{34} \mathbf{h} & j_{35} \mathbf{h} & j_{36} \mathbf{h} \\
j_{14} \mathbf{h}^{T} & j_{24} \mathbf{h}^{T} & j_{34} \mathbf{h}^{T} & j_{44} & j_{45} & j_{46} \\
j_{15} \mathbf{h}^{T} & j_{25} \mathbf{h}^{T} & j_{35} \mathbf{h}^{T} & j_{45} & j_{55} & j_{56} \\
j_{16} \mathbf{h}^{T} & j_{26} \mathbf{h}^{T} & j_{36} \mathbf{h}^{T} & j_{46} & j_{56} & j_{66}
\end{array}\right\}\left\{\begin{array}{c}
\mathbf{g}^{s} \\
\tilde{\mathbf{g}} \\
\mathbf{g}^{u} \\
\theta^{p} \\
\tilde{\theta} \\
\theta^{u}
\end{array}\right\} d V,
$$

where $I$ is the $6 \times 6$ identity matrix, $\mathbf{h}$ is defined in (49), and the derived fields are $\mathbf{s}^{g}=\mathbf{G} \tilde{\mathbf{g}}, \mathbf{s}^{u}=\mathbf{G}^{-1} \mathbf{D} \tilde{\mathbf{u}}, p^{\theta}=k \tilde{\theta}, p^{u}=-k \operatorname{div} \tilde{\mathbf{u}}, \mathbf{g}^{s}=\mathbf{G} \tilde{\mathbf{s}}, \mathbf{g}^{u}=\mathbf{G D} \tilde{\mathbf{u}}, \theta^{p}=k^{-1} \tilde{p}$ and $\theta^{u}=-\operatorname{div} \tilde{\mathbf{u}}$. In the incompressible limit $\theta^{p}$ vanishes. The analysis of [24] shows that consistency of the Euler-Lagrange equations with the field equations requires that the following nine constraints be satisfied:

$$
\begin{array}{lll}
j_{11}+j_{12}+j_{13}=0, & j_{14}+j_{15}+j_{16}=0, & j_{12}+j_{22}+j_{23}=0 \\
j_{24}+j_{25}+j_{26}=0, & j_{13}+j_{23}+j_{33}=1, & j_{34}+j_{35}+j_{36}=0 \\
j_{46}+j_{56}+j_{66}=1, & j_{44}+j_{45}+j_{46}=0, & j_{45}+j_{55}+j_{56}=0 .
\end{array}
$$

Furthermore, to include the incompressible limit, $j_{55}=j_{66}=0$. Consequently there are $21-11=10$ free parameters. The simplest generating matrix that satisfies all constraints is

$$
\mathbf{J}=\left[\begin{array}{llllll}
j_{11} & j_{12} & j_{13} & j_{14} & j_{15} & j_{16} \\
j_{12} & j_{22} & j_{23} & j_{24} & j_{25} & j_{26} \\
j_{13} & j_{23} & j_{33} & j_{34} & j_{35} & j_{36} \\
j_{14} & j_{24} & j_{34} & j_{44} & j_{45} & j_{46} \\
j_{15} & j_{25} & j_{35} & j_{45} & j_{55} & j_{56} \\
j_{16} & j_{26} & j_{36} & j_{46} & j_{56} & j_{66}
\end{array}\right]=\left[\begin{array}{cccccc}
0 & 0 & 0 & 0 & 0 & 0 \\
0 & 0 & 0 & 0 & 0 & 0 \\
0 & 0 & 1 & 0 & 0 & 0 \\
0 & 0 & 0 & -1 & 0 & 1 \\
0 & 0 & 0 & 0 & 0 & 0 \\
0 & 0 & 0 & 1 & 0 & 0
\end{array}\right] .
$$

This leads to a generalization of the PE functional (31) that exactly accomodates incompressibility. Other choices are examined in [24], where stress-strain splittings more general than (48) are considered. 


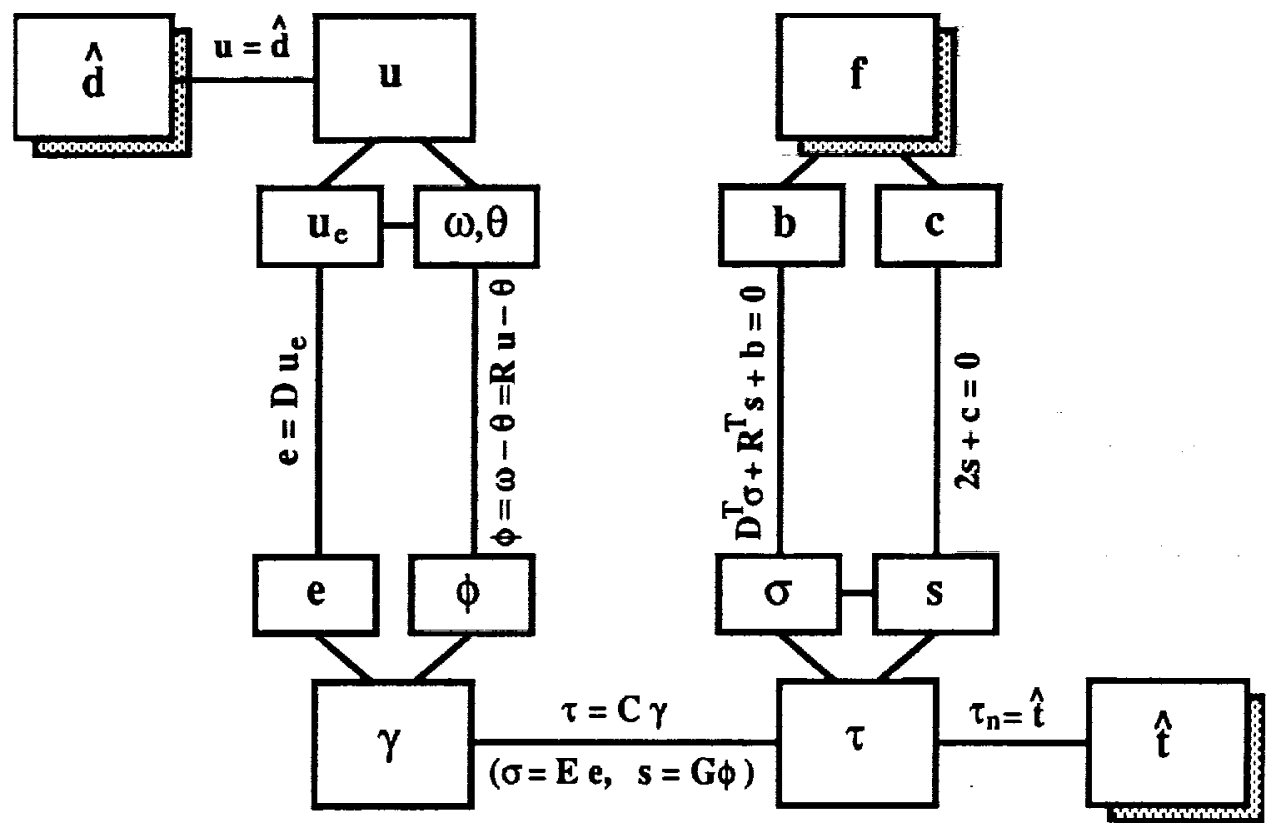

Fig. 9. Strong Form diagram for micropolar elastostatics without couple stresses.

\section{MICROPOLAR ELASTOSTATICS}

The extension to a linearly elastic micropolar medium without couple stresses has been investigated by Felippa [25]. In this elasticity model the stress tensor $\tau$ and the strain tensor $\gamma$ are no longer symmetric. They are decomposed as $\tau=\sigma+\mathbf{s}$, and $\gamma=\mathbf{e}+\phi$, where $\sigma$ and $\mathbf{e}$ are the symmetric parts and $\mathbf{s}$ and $\phi$ the antisymmetric parts, respectively. (Symbol $\mathbf{s}$ does not have the meaning of Section 6 .) General, symmetric and antisymmetric tensors that arise in this model may be arranged as 9,6, and 3-component vectors, respectively, to facilitate the use of matrix notation in the parametrized functionals. Notational details are given in that reference.

The rotational portion of $\mathbf{u}$ now enters the field equations. The infinitesimal rotation vector (also called macrorotations) is $\boldsymbol{\omega}=\mathbf{R u}$, where $\mathbf{R}$ is the rotor or curl operator. The microrotation vector $\theta$ is introduced so that the antisymmetric strains are given by $\phi=\boldsymbol{\omega}-\boldsymbol{\theta}$. The irrotational part of $\mathbf{u}$ is $\mathbf{u}_{e}$, which generates the symmetric strains $\mathbf{e}=\mathbf{D u}=\mathbf{D u}_{e}$, where $\mathbf{D}$ is again the symmetric gradient operator. Finally, body-volume actions are decomposed into $\mathbf{f}=\mathbf{b}+\mathbf{c}$, where $\mathbf{b}$ and $\mathbf{c}$ are the body force and body couple per unit volume, respectively. Classical elasticity is recovered if $\mathrm{c}$ vanishes.

The balance equations are $\mathbf{D}^{T} \boldsymbol{\sigma}+\mathbf{f}=0$ and $\mathbf{R}^{T} \mathbf{s}+\mathbf{c}=\mathbf{0}$. The constitutive equations are $\sigma=$ Ee and $\mathbf{s}=\mathbf{G} \phi$, which may be merged as $\boldsymbol{\tau}=\mathbf{C} \boldsymbol{\gamma}$, where $\mathbf{C}$ is block diagonal. These field equations, plus the boundary conditions $\mathbf{u}=\widehat{\mathbf{d}}$ on $S_{d}$ and $\tau_{n}=\widehat{\mathbf{t}}$ on $S_{t}$, are summarized in the Strong Form diagram of Figure 9.

The functional $\Pi$ again decomposes into $U-P$. The parametrized form of the internal 
energy $U$ has the structure

$$
U(\tilde{\mathbf{u}}, \tilde{\boldsymbol{\sigma}}, \tilde{\mathbf{s}}, \tilde{\mathbf{e}}, \tilde{\phi})=\frac{1}{2} \int_{V}\left\{\begin{array}{c}
\tilde{\boldsymbol{\sigma}} \\
\boldsymbol{\sigma}^{\boldsymbol{e}} \\
\boldsymbol{\sigma}^{u} \\
\tilde{\mathbf{s}} \\
\mathbf{s}^{\phi} \\
\mathbf{s}^{u}
\end{array}\right\}{ }^{T}\left[\begin{array}{cccccc}
j_{11} \mathbf{I}_{6} & j_{12} \mathbf{I}_{6} & j_{13} \mathbf{I}_{6} & \mathbf{0} & 0 & \mathbf{0} \\
j_{12} \mathbf{I}_{6} & j_{22} \mathbf{I}_{6} & j_{23} \mathbf{I}_{6} & 0 & 0 & 0 \\
j_{13} \mathbf{I}_{6} & j_{23} \mathbf{I}_{6} & j_{33} \mathbf{I}_{6} & \mathbf{0} & \mathbf{0} & \mathbf{0} \\
\mathbf{0} & \mathbf{0} & \mathbf{0} & j_{44} \mathbf{I}_{3} & j_{45} \mathbf{I}_{3} & j_{46} \mathbf{I}_{3} \\
\mathbf{0} & \mathbf{0} & \mathbf{0} & j_{45} \mathbf{I}_{3} & j_{55} \mathbf{I}_{3} & j_{56} \mathbf{I}_{3} \\
\mathbf{0} & \mathbf{0} & \mathbf{0} & j_{46} \mathbf{I}_{3} & j_{56} \mathbf{I}_{3} & j_{66} \mathbf{I}_{3}
\end{array}\right]\left\{\begin{array}{c}
\mathbf{e}^{\sigma} \\
\tilde{\mathbf{e}} \\
\mathbf{e}^{u} \\
\boldsymbol{\phi}^{s} \\
\tilde{\phi} \\
\phi^{u \theta}
\end{array}\right\} d V
$$

where $I_{6}$ and $I_{3}$ denote the identity matrices of order 6 and 3 , respectively. The derived quantities are $\sigma^{e}=\mathbf{E} \tilde{e}, \sigma^{u}=\mathbf{E D} \tilde{\mathbf{u}}, \mathbf{s}^{\phi}=\mathbf{G} \phi, \mathbf{s}^{u \theta}=\mathbf{G}(\mathbf{R} \tilde{\mathbf{u}}-\tilde{\theta}), \phi^{s}=\mathbf{G}^{-1} \tilde{\mathbf{s}}$ and $\phi^{u \theta}=\mathbf{R} \tilde{\mathbf{u}}-\tilde{\theta}$.

The zero entries of the kernel matrix in (54) reflect the orthogonality of symmetric and antisymmetric parts. The analysis carried out in [25] shows that consistency with the field equations requires that

$$
\begin{array}{lll}
j_{11}+j_{12}+j_{13}=0, & j_{12}+j_{22}+j_{23}=0, & j_{13}+j_{23}+j_{33}=1, \\
j_{35}+j_{45}+j_{46}=0, & j_{45}+j_{55}+j_{56}=0, & j_{46}+j_{56}+j_{66}=1 .
\end{array}
$$

Consequently there are $12-6=6$ free parameters in (54). One possible choice for the generating matrix that meets these constraints is

$$
\mathbf{J}=\left[\begin{array}{cccccc}
j_{11} & j_{12} & j_{13} & 0 & 0 & 0 \\
j_{12} & j_{22} & j_{23} & 0 & 0 & 0 \\
j_{13} & j_{23} & j_{33} & 0 & 0 & 0 \\
0 & 0 & 0 & j_{44} & j_{45} & j_{46} \\
0 & 0 & 0 & j_{45} & j_{55} & j_{56} \\
0 & 0 & 0 & j_{46} & j_{56} & j_{66}
\end{array}\right]=\left[\begin{array}{cccccc}
-1 & 0 & 1 & 0 & 0 & 0 \\
0 & 0 & 0 & 0 & 0 & 0 \\
1 & 0 & 0 & 0 & 0 & 0 \\
0 & 0 & 0 & -1 & 0 & 1 \\
0 & 0 & 0 & 0 & 0 & 0 \\
0 & 0 & 0 & 1 & 0 & 0
\end{array}\right]
$$

An alternative application of these functionals is to non-polar media in which $\mathbf{c}=\mathbf{0}$ although the symmetry of the stress tensor is only enforced weakly. Such functionals furnish a basis for construction of finite elements for classical elasticity with independently varied rotation fields, a subject that has recently been given impetus by the paper of Hughes and Brezzi [33]. In fact the choice (56) yields one of the canonical functionals derived in that paper. The Appendix of [25] extends these PVPs to micropolar media with couple stresses.

\section{ELECTROMAGNETODYNAMICS}

As final example, consider classical electromagnetodynamics based on Maxwell's equations. In this model the unknown internal fields are the magnetic and electric fluxes $H$, and $\mathbf{D}$ the magnetic and electric intensities $\mathbf{B}$ and $\mathbf{E}$, the magnetic potential $\mathbf{A}$ and the electric potential $\Phi$. The prescribed volume fields are the current intensity $\mathbf{j}$ and the electric source 


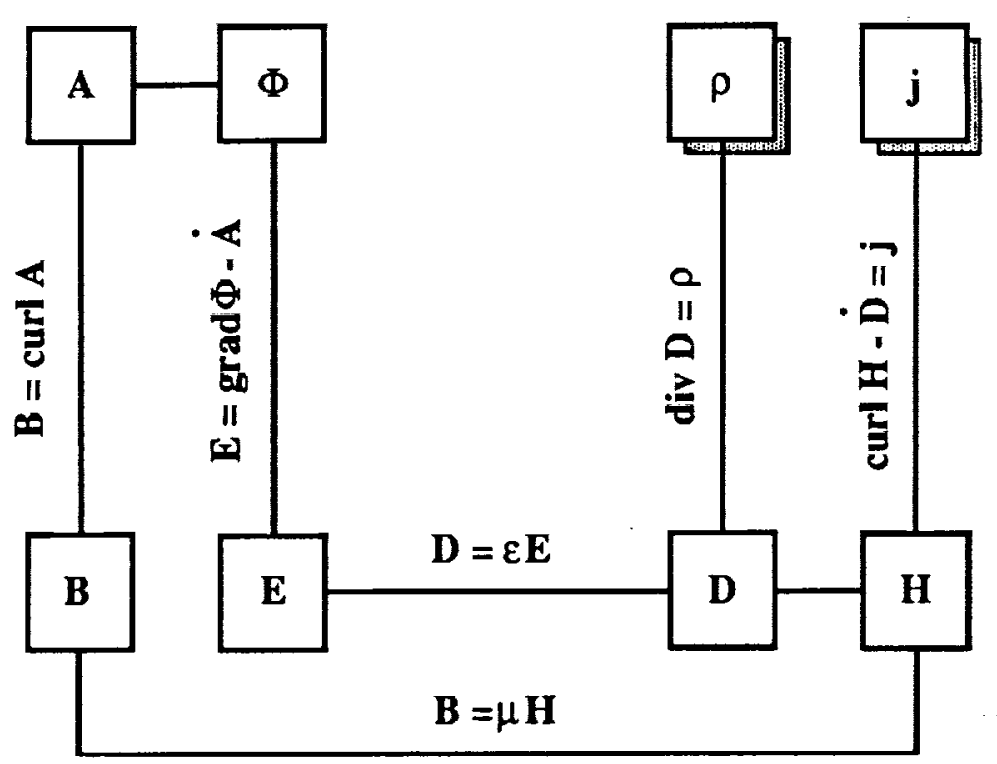

Fig. 10. The Strong Form of isotropic linear electromagnetodynamics, with boundary conditions omitted for simplicity. Here $\epsilon$ and $\mu$ denote the electric permittivity and magnetic permeability, respectively, of the medium. Other symbols are defined in the text. A superposed dot denotes derivative with respect to time $t$.

p. The field equations are summarized in the SF depicted in Figure 10, in which boundary conditions have been omitted for simplicity.

The general functional has the form $\Pi=U-S-B$, where $U$ is the generalized electromagnetic energy, $S$ includes source terms and $B$ contains boundary-closure terms. A parametrized form of $U$ studied by Felippa and Schuler [28] is

$$
U=\frac{1}{2} \int_{V \times t}\left\{\begin{array}{c}
c^{-1} \tilde{\mathbf{H}} \\
c^{-1} \mathbf{H}^{B} \\
c^{-1} \mathbf{H}^{A} \\
\tilde{\mathbf{D}} \\
\mathbf{D}^{E} \\
\mathbf{D}^{\Phi A}
\end{array}\right\}\left[\begin{array}{llllll}
g_{11} \mathbf{I} & g_{12} \mathbf{I} & g_{13} \mathbf{I} & g_{14} \mathbf{I} & g_{15} \mathbf{I} & g_{16} \mathbf{I} \\
g_{12} \mathbf{I} & g_{22} \mathbf{I} & g_{23} \mathbf{I} & g_{24} \mathbf{I} & g_{25} \mathbf{I} & g_{26} \mathbf{I} \\
g_{13} \mathbf{I} & g_{23} \mathbf{I} & g_{33} \mathbf{I} & g_{34} \mathbf{I} & g_{35} \mathbf{I} & g_{36} \mathbf{I} \\
g_{14} \mathbf{I} & g_{24} \mathbf{I} & g_{34} \mathbf{I} & g_{44} \mathbf{I} & g_{45} \mathbf{I} & g_{46} \mathbf{I} \\
g_{15} \mathbf{I} & g_{25} \mathbf{I} & g_{35} \mathbf{I} & g_{45} \mathbf{I} & g_{55} \mathbf{I} & g_{56} \mathbf{I} \\
g_{16} \mathbf{I} & g_{26} \mathbf{I} & g_{36} \mathbf{I} & g_{46} \mathbf{I} & g_{56} \mathbf{I} & g_{66} \mathbf{I}
\end{array}\right]\left\{\begin{array}{c}
c \mathbf{B}^{H} \\
c \tilde{\mathbf{B}} \\
c \mathbf{B}^{A} \\
\mathbf{E}^{D} \\
\tilde{\mathbf{E}} \\
\mathbf{E}^{\Phi A}
\end{array}\right\} d V d t
$$

where $I$ is the $3 \times 3$ identity matrix and $c$ is the speed of propagation of EM disturbances. (The parametrization coefficients for this functional are denoted by $g$ instead of $j$ to avoid confusion with the symbol for current density.) The derived terms in (57) are $\mathbf{H}^{B}=\mu^{-1} \tilde{\mathbf{B}}$, $\mathrm{H}^{A}=\mu^{-1}$ curl $\tilde{\mathbf{A}}, \mathbf{D}^{E}=\epsilon \tilde{\mathbf{E}}$, etc. The analysis of [28] shows that the $g$ coefficients must 
satisfy the 12 consistency constraints:

$$
\begin{array}{lcc}
g_{11}+g_{12}+g_{13}=0, & g_{14}+g_{15}+g_{16}=0 \\
g_{12}+g_{22}+g_{23}=0, & g_{24}+g_{25}+g_{26}=0 \\
g_{13}+g_{23}+g_{33}=1, & g_{16}+g_{26}+g_{36}=0 \\
g_{34}+g_{35}+g_{36}=0, & g_{46}+g_{56}+g_{66}=-1 \\
g_{14}+g_{24}+g_{34}=0, & g_{44}+g_{45}+g_{46}=0 \\
g_{15}+g_{25}+g_{35}=0, & g_{45}+g_{55}+g_{56}=0
\end{array}
$$

which leaves $21-12=9$ free parameters. The simplest choice for the generating matrix is

$$
\mathbf{G}=\left[\begin{array}{lllllll}
g_{11} & g_{12} & g_{13} & g_{14} & g_{15} & g_{16} \\
g_{12} & g_{22} & g_{23} & g_{24} & g_{25} & g_{26} \\
g_{13} & g_{23} & g_{33} & g_{34} & g_{35} & g_{36} \\
g_{14} & g_{24} & g_{34} & g_{44} & g_{45} & g_{46} \\
g_{15} & g_{25} & g_{35} & g_{45} & g_{55} & g_{56}
\end{array}\right]=\left[\begin{array}{cccccc}
0 & 0 & 0 & 0 & 0 & 0 \\
0 & 0 & 0 & 0 & 0 & 0 \\
0 & 0 & 1 & 0 & 0 & 0 \\
0 & 0 & 0 & 0 & 0 & 0 \\
0 & 0 & 0 & 0 & 0 & 0 \\
0 & 0 & 0 & 0 & 0 & -1
\end{array}\right]
$$

which, on including prescribed sources $\rho$ and $\mathbf{j}$, yields the two-field Lagrangian

$$
\int_{t_{1}}^{t_{2}} \int_{V}\left\{\frac{1}{2} \mu^{-1}\|\nabla \times \tilde{\mathbf{A}}\|^{2}-\frac{1}{2} \epsilon\left\|\nabla \tilde{\Phi}+\frac{\partial \tilde{\mathbf{A}}}{\partial t}\right\|^{2}-\mathbf{j}^{T} \tilde{\mathbf{A}}-\rho \tilde{\Phi}\right\} d V d t .
$$

Felippa and Schuler [28] extend this PVP to the situation in which the current density $\mathbf{j}$ is unknown, in which case Ohm's law has to be adjoined to the governing equations. This extension is important as a departure point for the finite element analysis of Type I and II superconductors.

\section{APPLICATIONS TO FINITE ELEMENT METHODS}

From previous examples it is seen that multifield PVPs are interesting in their own right because they provide a continuous space of functionals. Canonical functionals are "instance points" of this space. Moving in this space is equivalent to changing weights on the field equations. A theorem proven for a parametrized functional is "economical" in the sense that it need not be redone for specific instances.

Traditionally books dealing with applications of variational calculus in mechanics jump from one specific canonical functional to another. This approach has two instructional disadvantages. First the reader is left with the impression that only a finite number of functionals exist. Second, what ought to be shared properties are proved over and over, a boring repetitive feat that can easily span hundreds of pages.

Aside from this theoretical and educational unifying value, multifield PVPs offer applications to variationally-based methods of approximation in general and the Finite Element Method (FEM) in particular. Following are three intriguing subjects pertaining to FEM, which in the author's opinion merit further exploration. 
(A) Improved Approximation. Approximate FEM solutions generally depend on the free parameters while the converged solution (assuming a convergent approximation method) does not. Can the parameters be chosen to improve the quality of approximation on a fixed finite element mesh?

(E) A-posteriori error estimation. Assume the PVP is an IPVP. Suppose we obtain FE solutions for two different parameter sets on the same mesh. As the mesh is refined the values of the functional for both solutions approaches each other over each element. Can this property be exploited to construct local (element level) error estimators?

(T) Templates. Using PVPs, can we derive universal "templates" for FE matrix expressions that covers all convergent finite elements of a given freedom configuration?

The answers to these questions are in different state of development.

Exploiting (A) leads to high-performance finite elements. This is an application that has been investigated over the past four years with focus in classical elasticity, and has led to production-level finite elements. Knowledge as to the effect of parameter selection is increasing, but much remains to be done.

Exploiting (E) leads to element-level error estimators. Only limited numerical experimentation has been carried out, and most of the theory remains to be developed.

The idea of templates $(\mathrm{T})$ has been explored for some specific finite elements, but it remains largely a conjecture.

The next three Sections describe the state of these applications in further detail.

\section{APPLICATION 1: HIGH PERFORMANCE FINITE ELEMENTS}

A high performance finite element (HPFE for short) for structural mechanics is a simple element that delivers results of engineering accuracy on coarse meshes.

By "simple" it is meant that the element has few degrees of freedom, all physical, preferably at corners only. Engineering accuracy typically means less than $1 \%$ error in displacements and $5 \%$ in stresses. The term "coarse mesh" is subjective. In terms of structural applications, it characterizes a mesh that an experienced modeler would use to capture the basic physics with a minimum number of degrees of freedom. This number is of course problem dependent: a coarse mesh for a simple supported, uniform loaded plate would typically be a $2 \times 2$ or $4 \times 4$ mesh with less than 100 freedoms; on the other hand a coarse mesh for a complete aerospace vehicle may involve 50,000 to a million freedoms.

Mandatory attributes for a HPFE are: convergent, frame invariant and rank sufficient. Desirable attributes include:

- Yields similar accuracy in displacements and stresses.

- Is relatively insensible to geometric distortion.

- Is mixable with other elements.

- Provides effective a-posteriori error estimators to drive mesh adaptation procedures. 
- Fits naturally into displacement-based FE programs.

- Extends readily to nonlinear and dynamic problems.

The goal of attaining reasonable accuracy with coarse meshes of simple elements is the key one, however, and deserves further comment. This requirement should not be confused with fast asymptotic convergence for fine meshes. Simple elements cannot effectively compete with higher order elements in this regard, and are not effective in applications that demand very high accuracy. What matters is the accuracy obtained for the meshes typically used in complex engineering systems, as discussed above.

\subsection{Unification}

Many tools and techniques have been developed over the past three decades to develop HPFEs. The most practically important are: (a) incompatible shape functions; (b) hybrid and mixed formulations; (c) reduced, selective and directional numerical integration; (d) assumed natural strains and the Free Formulation. These four techniques originated in the mid 1960s, late 1960s, early 1970s and early 1980s, respectively.

The approach recently pursued by the author has relied upon the use of PVPs with hybrid treatment of element interfaces. More specifically, three ingredients are used:

1. The parametrized internal energy functional (41).

2. A $d$-generalized hybrid treatment of interfaces through the forcing potential (36).

3. Additional assumptions (sometimes called "variational crimes" or "tricks") that can be placed on a variational setting through Lagrange multipliers.

The first two ingredients says that HPFEs can be effectively constructed using hybrid PVPs of the form

$$
\Pi^{d}(\tilde{\mathbf{u}}, \tilde{\boldsymbol{\sigma}}, \tilde{\mathbf{e}}, \tilde{\mathbf{d}})=U(\tilde{\mathbf{u}}, \tilde{\boldsymbol{\sigma}}, \tilde{\mathbf{e}})-P^{d}(\tilde{\mathbf{u}}, \tilde{\boldsymbol{\sigma}}, \tilde{\mathbf{e}}, \tilde{\mathbf{d}}),
$$

where $U$ and $P^{d}$ are given by (41) and (36), respectively. Observe that $P^{d}$ contains independently varied displacements $\tilde{\mathbf{d}}$ on element interfaces. This allows the internal displacements $\tilde{\mathbf{u}}$ to be nonconforming and in some cases (ANDES elements) ignored entirely.

Throughout this section we shall deal with individual elements only. Element identifiers, which will be required in Section 11, are omitted for simplicity.

For mechanical elements modeled by classical elasticity, the HPFE derivation method summarized in Figure 11 leads to finite element stiffness equations that decompose as

$$
\left(\mathbf{K}_{b}+\mathbf{K}_{h}\right) \mathbf{v}=\mathbf{p}
$$

where $\mathbf{v}$ are visible degrees of freedom (those matched with other elements, also known as connectors), $\mathbf{p}$ are associated node forces, $\mathbf{K}_{b}$ is the basic stiffness matrix, which is constructed for convergence and mixability, and $\boldsymbol{K}_{h}$ is the higher order stiffness matrix, which is constructed for stability and accuracy. This decomposition was first obtained, without variational arguments, by Bergan and Nygård in the derivation of the unscaled Free Formulation [12]. A key result of the multifield variational formulation is that only $\mathbf{K}_{h}$ depends on the functional parameters $[22,23]$. 


\section{Element Basic Higher Order}

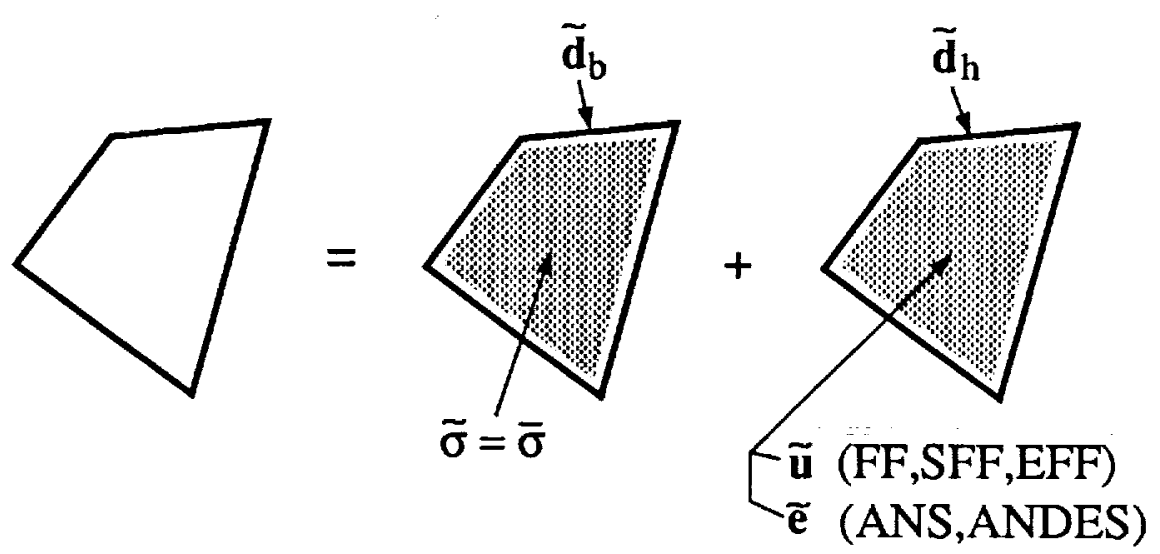

Fig. 11. Summary of assumptions made in constructing a high-performance mechanical element from the PVP (61). For the basic stiffness an interior constant stress field $\tilde{\boldsymbol{\sigma}}=\overline{\boldsymbol{\sigma}}$ and an interelement-compatible boundary displacement field $\tilde{\mathbf{d}}_{b}$ are assumed. For the higher order stiffness either an internal displacement field $\tilde{\mathbf{u}}$ is assumed with the FF and its variants, or an internal strain field $\tilde{e}$ is assumed with the ANS formulation and its variants. (Only the deviatoric part of appears in the ANDES variant.) The boundary displacement field $\tilde{\mathbf{d}}_{h}$ need not be the same as $\tilde{d}_{b}$, although it often is. In the FF and variants thereof, $\tilde{\mathbf{d}}_{h}$ and $\tilde{\mathbf{u}}$ are collocated at node points.

\subsection{The Parametrized 3-Node Bar Element}

The 3-node bar element depicted in Figure 12 will be used to illustrate the steps in the derivation of HPFE stiffness equations from PVPs. The element has total length $2 h$ and constant axial rigidity $E A$. The applied force per unit length is $f(x)$. The internal fields are the axial displacement $u(x)$, axial strain $e(x)=u^{\prime}(x)$, and internal axial force $N(x)=A \sigma(x)=E A e(x)$. The 3 degrees of freedom (dof) are the node displacements $v_{1}$, $v_{2}$ and $v_{3}$ along the bar axis. For an internal finite element of this type, the $d$-generalized parametrized functional (61) reduces to

$$
\begin{aligned}
\Pi^{d}(\tilde{u}, \tilde{N}, \tilde{e}, \tilde{d})= & \frac{1}{2} \int_{-h}^{h}\left\{\begin{array}{c}
\tilde{N} \\
N^{e} \\
N^{u}
\end{array}\right\}{ }^{T}\left[\begin{array}{ccc}
j_{11} & -s_{3} & -s_{2} \\
-s_{3} & j_{22} & -s_{1} \\
-s_{2} & -s_{1} & j_{33}
\end{array}\right]\left\{\begin{array}{c}
\tilde{e}^{N} \\
\tilde{e} \\
e^{u}
\end{array}\right\} d x-\int_{-h}^{h} f u d x \\
& -\left.\dot{N}(\tilde{d}+\tilde{u})\right|_{-h}-\left.\dot{N}(\tilde{d}-\tilde{u})\right|_{h}
\end{aligned}
$$

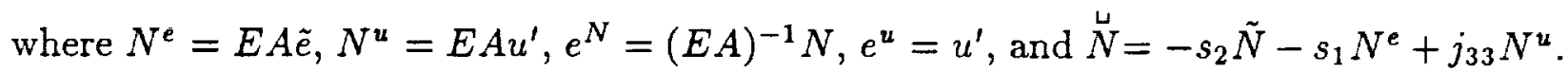
To construct the element we make assumptions on the varied fields $\tilde{N}, \tilde{u}, \tilde{e}$ and $\tilde{d}$. In expressing $\tilde{u}$ and $\tilde{e}$ it is convenient to introduce the hierarchical displacement of node 2: $\check{v}_{2}=v_{2}-\frac{1}{2}\left(v_{1}+v_{3}\right)$, which is the midpoint deviation from linearity. We also introduce the natural coordinates $\zeta_{1}=\frac{1}{2}(1-x / h)$ and $\zeta_{3}=\frac{1}{2}(1+x / h)$ for convenience. 


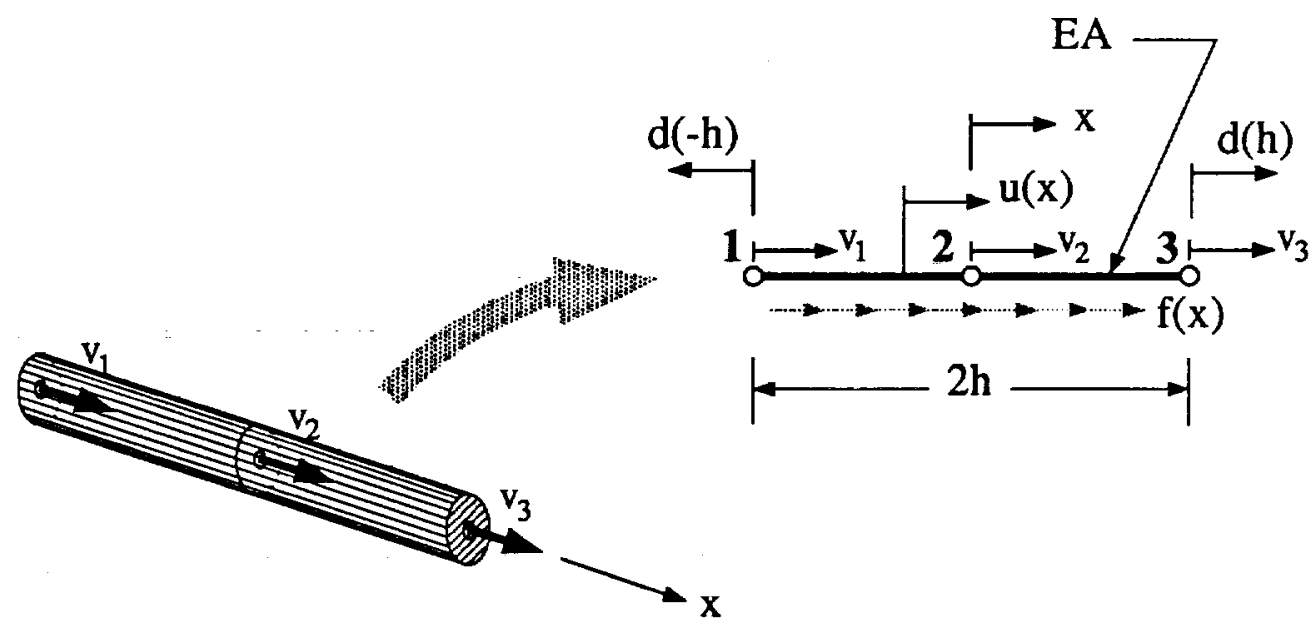

Fig. 12. Three-node bar element used as example of derivation of stiffness equations from the $d$-generalized principle (63).

The internal force is constant over the element: $\tilde{N}=\bar{N}$. The mean force $\bar{N}$ is a degree of freedom that (thanks to the hybrid treatment) can be eliminated at the element level.

The axial displacement is expanded into a basic (linearly-varying) component $u_{b}$, which represents rigid body modes and constant strain states, and a higher-order (quadraticallyvarying) component $u_{h}$ :

$$
\tilde{u}(x)=u_{b}(x)+u_{h}(x), \quad u_{b}(x)=v_{1} \zeta_{1}+v_{3} \zeta_{3}, \quad u_{h}(x)=4 \check{v}_{2} \zeta_{1} \zeta_{3} .
$$

The assumed strain is also split into a basic component (the average or mean strain $\bar{e}$ ) and a higher-order component called the deviatoric strain:

$$
\tilde{e}=\bar{e}+e_{h}, \quad \bar{e}=\left(v_{3}-v_{1}\right) / h, \quad e_{h}(x)=\frac{\check{v}_{2}}{h}\left[\mu \operatorname{sign}(x)-2(1-\mu) \frac{x}{h}\right]
$$

Observe that $e_{h}$ is represented here by a constant-plus-linear function that is odd in $x$, and contains a coefficient $\mu$ that weights the "mixing" between the constant and linear parts. If $\mu=0$ the strain varies linearly as in the quadratic-displacement element and $\tilde{e}=e^{u}$. If $\mu=1$ the strain is constant over subelements $1-2$ and 2-3 and agrees with that of the "macroelement" constructed with two linear 2-node bar elements. The assumption (65) satisfies the compatibility conditions $\int_{-h}^{0} e_{h}(x) d x=-\int_{0}^{h} e_{h}(x) d x=\check{v}_{2}$ for any $\mu$.

Finally, the boundary displacements are identified with the end-node displacements:

$$
\tilde{d}(-h)=\left.\tilde{d}\right|_{-h}=-v_{1}, \quad \tilde{d}(h)=\left.\tilde{d}\right|_{h}=v_{3} .
$$

Because $u(x)$ also agrees exactly with $v_{1}$ and $v_{3}$ at the end nodes, the boundary term in (63) vanishes. Inserting the above assumptions into (63) produces a quadratic form in the degrees of freedom $v_{1}, \check{v}_{2}, v_{3}$ and $\bar{N}$. Rendering the form stationary with respect to 
these freedoms leads to a system of linear equations that immediately gives $\bar{N}=E A \bar{e}=$ $E A\left(v_{3}-v_{1}\right) / h$. Elimination of $\bar{N}$ yields the element stiffness equations

$$
\left(\frac{E A}{2 h}\left[\begin{array}{ccc}
1 & 0 & -1 \\
0 & 0 & 0 \\
-1 & 0 & 1
\end{array}\right]+\beta \frac{2 E A}{h}\left[\begin{array}{lll}
0 & 0 & 0 \\
0 & 1 & 0 \\
0 & 0 & 0
\end{array}\right]\right)\left\{\begin{array}{l}
v_{1} \\
\check{v}_{2} \\
v_{3}
\end{array}\right\}=\left\{\begin{array}{l}
p_{1} \\
\check{p}_{2} \\
p_{3}
\end{array}\right\}
$$

where forces $p_{1}, \check{p}_{2}$ and $p_{3}$ result from the nodal lumping of $f(x)$ and

$$
\beta=\frac{1}{3}\left[(4-\mu) j_{11}+\left(\mu^{2}-\mu\right) j_{22}+\mu j_{33}+(4-\mu)\right]=\frac{1}{3}\left[\mu^{2} s_{1}+4 s_{2}+\left(\mu^{2}-2 \mu+4\right) s_{3}+4\right] .
$$

Expressing (67) in terms of the total midpoint displacement $v_{2}$ rather than $\check{v}_{2}$ yields

$$
\begin{aligned}
\mathbf{K} \mathbf{v}=\left(\mathbf{K}_{b}+\mathbf{K}_{h}\right) \mathbf{v} & =\left(\frac{E A}{2 h}\left[\begin{array}{ccc}
1 & 0 & -1 \\
0 & 0 & 0 \\
-1 & 0 & 1
\end{array}\right]+\beta \frac{E A}{2 h}\left[\begin{array}{ccc}
1 & -2 & 1 \\
-2 & 4 & -2 \\
1 & -2 & 1
\end{array}\right]\right)\left\{\begin{array}{l}
v_{1} \\
v_{2} \\
v_{3}
\end{array}\right\} \\
& =\frac{E A}{6 h}\left[\begin{array}{ccc}
3+3 \beta & -6 \beta & -3+3 \beta \\
-6 \beta & 12 \beta & -6 \beta \\
-3+3 \beta & -6 \beta & 3+3 \beta
\end{array}\right]\left\{\begin{array}{l}
v_{1} \\
v_{2} \\
v_{3}
\end{array}\right\}=\left\{\begin{array}{l}
p_{1} \\
p_{2} \\
p_{3}
\end{array}\right\}
\end{aligned}
$$

Here we see the emergence of the stiffness decomposition (62). The basic stiffness $\mathbf{K}_{b}$ contains no parameters and has rank one, so by itself it is rank deficient. The higher order stiffness has also rank one and its addition stabilizes $\mathbf{K}$ as long as $\beta>0$.

Although the FEM solution converges for any positive $\beta$, maximum accuracy in smooth problems is obtained for $\beta=4 / 3$. This yields the well known 3-node quadratic bar element. [To check this, take $j_{33}=1, j_{11}=j_{22}=0$ in (68) to get the PE functional, which annihilates the separate strain assumption (65).] On the other hand, setting $\beta=1$ yields the 3-node "macroelement" equivalent to assembling two linear bar elements. [To check this, take $j_{22}=1, j_{11}=j_{33}=0$ in (68) to get the HW functional and set $\mu=1$ ].

The foregoing derivation appears to be overkill for such a simple element. Indeed (69) can be obtained without recourse to any variational principle. Direct physical reasoning (as in the original $\mathrm{FF}$ ), algebraic arguments relying on subspace decomposition of node motions, or even finite differences: all methods lead to (69). Such universality is the motivation behind the template conjecture discussed in Section 12. In two and three dimensions and especially complicated elements, however, variationally-based methods do have a place.

\subsection{The Parametrized 2-Node Plane Beam Element}

As a second one-dimensional example consider a two-node element for an Euler-Bernouilli $\left(C^{1}\right)$ plane beam of constant rigidity $E I$ and span $h$. The four nodal degrees of freedom are the transverse end displacements $w_{1}$ and $w_{2}$ and the end rotations $\theta_{1}$ and $\theta_{2}$ as shown in Figure 14. Any parametrization method, whether variationally based or not, produces 


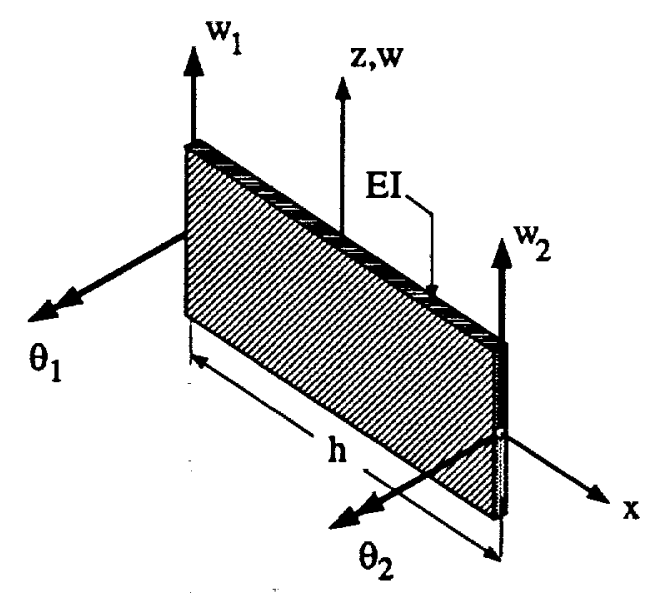

Fig. 13. Two-node Euler-Bernouilli beam element.

a decomposed stiffness matrix of the form

$$
\mathbf{K}=\mathbf{K}_{b}+\mathbf{K}_{h}=\frac{E I}{h}\left[\begin{array}{cccc}
0 & 0 & 0 & 0 \\
0 & 1 & 0 & -1 \\
0 & 0 & 0 & 0 \\
0 & -1 & 0 & 1
\end{array}\right]+\beta \frac{E I}{h^{3}}\left[\begin{array}{cccc}
4 & -2 h & -4 & -2 h \\
-2 h & h^{2} & 2 h & h^{2} \\
-4 & 2 h & 4 & 2 h \\
-2 h & h^{2} & 2 h & h^{2}
\end{array}\right]
$$

where $\beta>0$. Both $\mathbf{K}_{b}$ and $\mathbf{K}_{h}$ have rank one, and combine to provide the proper rank of two. The (optimal) Hermitian-cubic beam element is recovered if $\beta=3$.

An interesting property is that the Timoshenko $\left(C^{0}\right)$ beam has exactly the same stiffness decomposition but the optimal $\beta$ is then $3+36(E I / G A) h^{-2}$, where $G A$ is the sectionaveraged shear rigidity; if $G A \rightarrow \infty$ or $h \rightarrow 0, \beta \rightarrow 3$. The distinction made in the copious finite element literature on $C^{0}$ versus $C^{1}$ beam elements is seen to be artificial.

\subsection{Three High-Performance Triangles}

For multidimensional elements the general parametrized functional (61) has not been yet exploited in its full generality. As of this writing only two one-parameter subsets have been studied in some detail:

1. A stress-displacement $d$-generalized functional $\Pi_{\gamma}^{d}(\tilde{\mathbf{u}}, \tilde{\boldsymbol{\sigma}}, \tilde{\mathrm{d}})$ associated with the Free Formulation (FF) and two variants thereof: the Scaled Free Formulation (SFF) introduced in [13] and the Extended Free Formulation (EFF) proposed in [21]. Its free parameter is called $\gamma$. Reduces to PE for $\gamma=0$ and to HR for $\gamma=1$ (see Figure 14).

2. A stress-strain-displacement $d$-generalized functional $\Pi_{\alpha}^{d}(\tilde{\mathbf{u}}, \tilde{\boldsymbol{\sigma}}, \tilde{\mathbf{e}}, \tilde{\mathbf{d}})$ associated with the Assumed Natural Deviatoric Strain (ANDES) formulation. Its free parameter is called $\alpha$. Reduces to HR for $\alpha=0$ and HW for $\alpha=1$ (see Figure 14).

The most successful multidimensional HP elements constructed to date using these subsets are depicted in Figure 15 and briefly described below. The EFFAND element is a plane stress triangle (membrane component in shell element) with nine degrees of freedom, three 


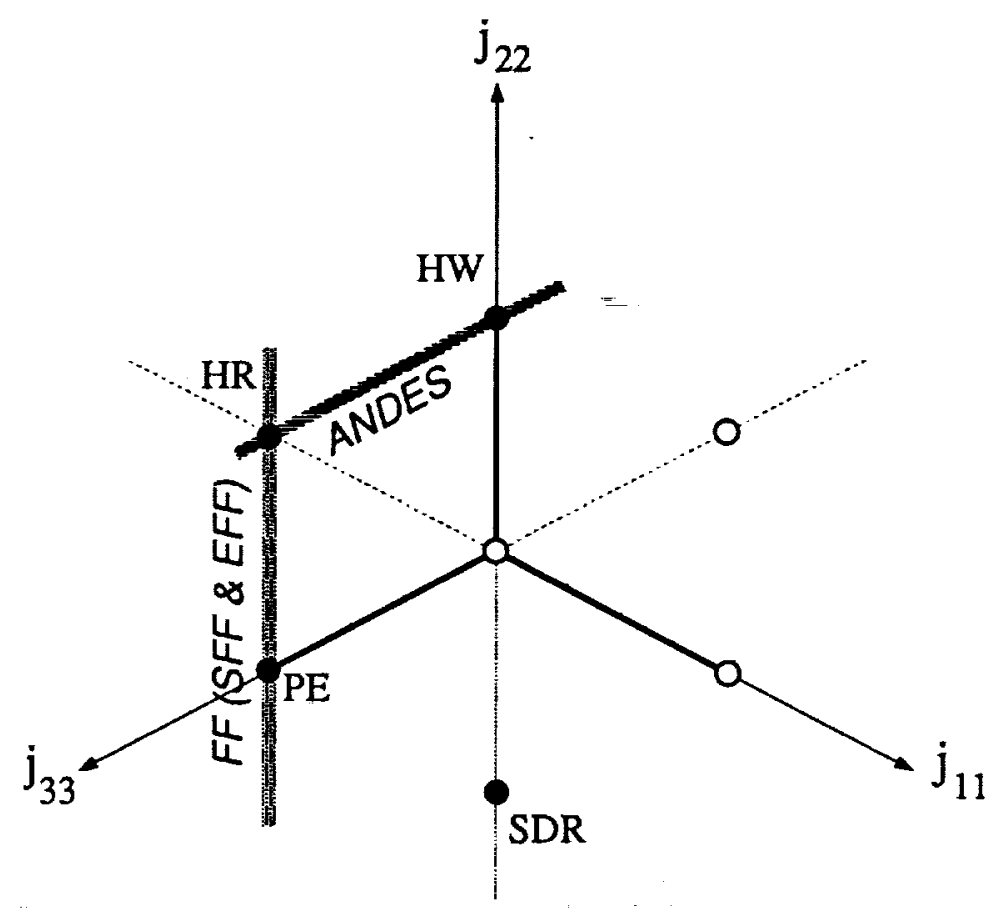

Fig. 14. The one-parameter functionals associated with the Free Formulation $(\mathrm{FF})$ and Assumed Natural Deviatoric Strain (ANDES) formulation of finite elements, depicted in the general $\left(j_{11}, j_{22}, j_{33}\right)$ space of Figure 6.

of which are corners "drilling" rotations $\theta_{z}$. It represents the culmination of a development began by Bergan and Felippa [13] with the Scaled Free Formulation. The latest element was derived with both the Extended Free Formulation [21] and the Assumed Natural Deviatoric Strain (ANDES) formulation $[2,26,27]$. Triangles optimized for bending behavior were found to coalesce, and this optimal element named EFFAND.

The insensitivity of EFFAND with respect to element aspect ratio is illustrated in Table 2, which pertains to the beam-under-pure-bending test problem shown in Figure 16. The results of Table 2 are taken from Felippa and Alexander [27]. In this Table ALL- $x x$ denote three numerically integrated versions of the 1987 Allman rank-sufficient triangle [1], CST is the constant strain triangle [61], and FF is the first-generation triangle of this type constructed by Bergan and Felippa [13].

AQR is a Kirchhoff (thin) plate bending element constructed by Militello and Felippa [43]. The element has the standard nine degrees of freedom and is based on the ANDES formulation. An extensive set of numerical tests shows that AQR generally outperforms other elements of this type tested to date as regards distortion sensitivity.

Finally, the EFFAND-AQR shell element is a combination of EFFAND (membrane component) and AQR (bending component) to form a triangular-facet shell element. It has 18 degrees of freedom: 9 for membrane plus 9 for bending. This element has been found to excel in modeling complete aerospace vehicles for stiffness and vibration analysis because:

(a) Membrane/bending accuracy is balanced. 


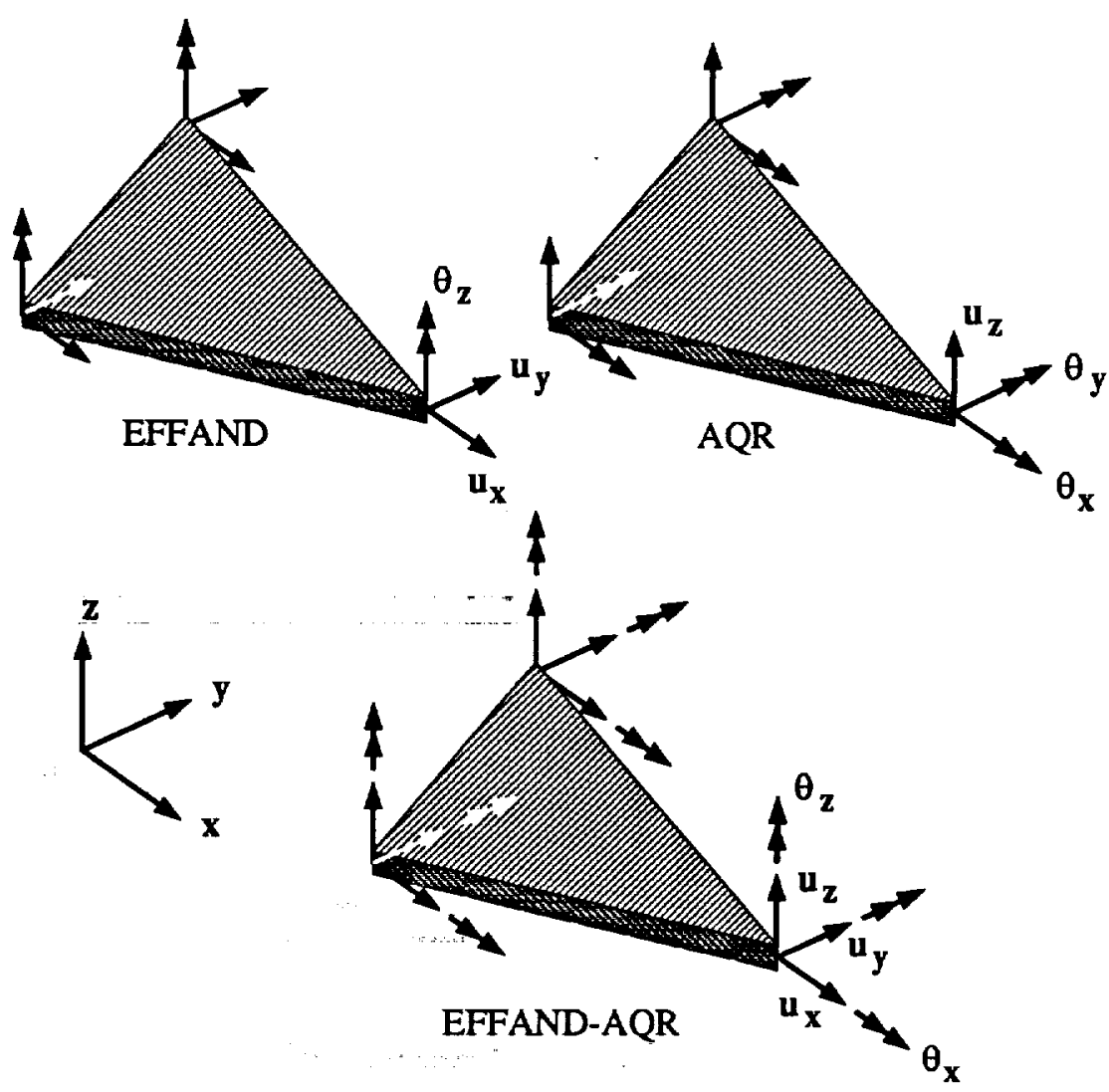

Fig. 15. Three high-performance elements constructed with PVPs: the EFFAND membrane 9-dof element, the AQR plate-bending 9-dof element, and the EFFAND-AQR 18-dof shell element.

(b) Global results - e.g. frequencies - of engineering accuracy may be obtained with "coarse" meshes (for a complete aircraft, 50,000 to 200,000 dof). This is important for full-vehicle aeroelastic simulations.

(c) The presence of a complete set of rotational freedoms eliminates juncture modeling problems.

(d) The element simplicity helps implementation on medium and fine-grained parallel supercomputers with limited memory per processor.

\section{APPPLICATION 2: LOCAL ERROR ESTIMATION}

As second PVP application we take up the subject of a posteriori estimation of the local discretization error of a computed finite element solution. These estimators may be used to guide computer-driven mesh adaptation processes based on $r, h$ or $p$ techniques.

The theory of FE local estimation and mesh adaptivity has received substantial attention over the past 17 years since the appearance of the original papers by Babuška [4] and Sewell [56]. Much of the theory pertains to conforming finite element models based on the Potential Energy principle or its equivalents. Because elements based on these assumptions 

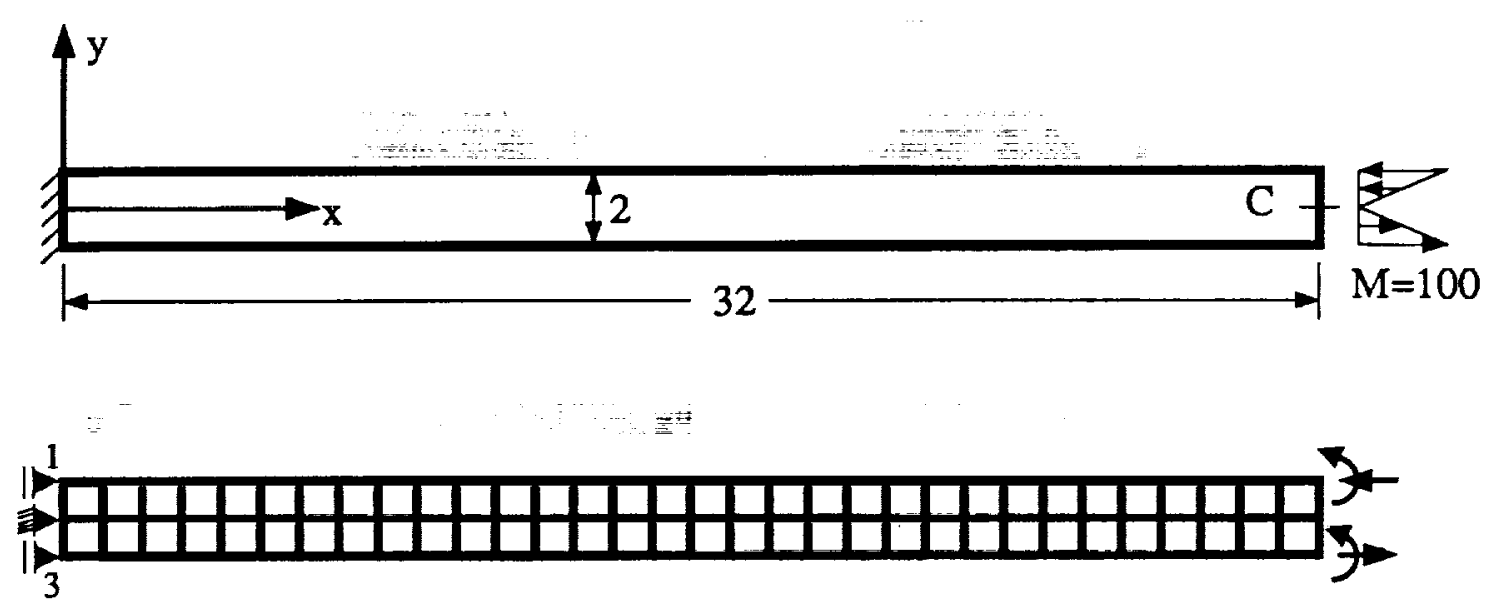

Fig. 16. Test problem for high-performance membrane element: 16:1 cantilever of rectangular cross section under pure end moment $M$. Poisson's ratio $\nu=0, E$ adjusted so that exact tip deflection is 100. A $32 \times 2$ mesh is shown. Each rectangular macroelement is made up of four "crisscrossed" membrane triangles. (After [27].)

Table 2. Tip Deflections (exact=100) for Beam of Figure 16

\begin{tabular}{|lrrrrrr|}
\hline Element & $\nu$ & \multicolumn{5}{c|}{ Mesh: $x$-subdivisions $\times y$-subdivisions } \\
& & $32 \times 2$ & $16 \times 2$ & $8 \times 2$ & $4 \times 2$ & $2 \times 2$ \\
\hline ALL-3i & 0 & 87.99 & 75.47 & 37.01 & 5.51 & 0.42 \\
ALL-3m & 0 & 81.02 & 51.62 & 9.64 & 0.74 & 0.04 \\
ALL-7i & 0 & 85.43 & 67.44 & 23.65 & 2.55 & 0.17 \\
CST & 0 & 53.33 & 33.33 & 13.33 & 3.92 & 1.02 \\
EFFAND & 0 & 100.00 & 100.00 & 100.00 & 100.00 & 100.00 \\
FF & 0 & 100.25 & 99.15 & 98.38 & 98.08 & 97.98 \\
\hline
\end{tabular}

are rarely the best performing ones, a curious situation arises in which the well developed error theory applies to rather uninteresting elements.

\subsection{Error Equations for Poisson's Problem}

We review the basic error equations for a conforming finite element discretization of the Poisson's problem discussed in Section 3.2 based on the PE-like principle (14). The analysis follows essentially Babuška and Miller [5] as well as Babuška, Durán and Rodriguez [6].

The bounded domain $\Omega$ with boundary $\Gamma$ is replaced by a regular FE mesh with nonoverlapping elements numbered $e=1, \ldots N^{e}$. Element $e$ has interior $\Omega^{e}$ and boundary $\Gamma^{e}$. The latter is composed of $N_{s}^{e}$ element sides identified as $\Gamma_{j}^{e}, j=1, \ldots N_{s}^{e}$. For simplicity the geometric discretization error is neglected so that $\Omega \equiv \cup_{e} \Omega^{e}$ and $\Gamma \equiv \cup_{e, j} \Gamma_{j}^{e} \forall \Gamma_{j}^{e} \in \Gamma$. Further it is assumed that when an element side belongs to $\Gamma$, it is completely contained 
in either $\Gamma_{u}$ or $\Gamma_{q}$. The union of element boundaries $\Gamma_{j}^{e}$ not on $\Gamma$ is called $\Gamma_{i}$, which thus collects all internal sides. An internal side that is shared by two adjacent elements $e$ and $d$ is called $\Gamma^{e d}$.

Let $u=u(x, y)$ be the exact solution of the Poisson problem (11)-(12) with $f \in L^{2}(\Omega)$, $\widehat{q} \in L^{2}\left(\Gamma_{q}\right)$ and $\hat{u}=0$ on $\Gamma_{d}$. Let $\tilde{u}=\tilde{u}(x, y) \in H^{1}(\Omega): \Gamma_{d}=0$ be a conforming finite element solution, and $\epsilon^{u}=u-\tilde{u}$ the approximation error. The localization of $\tilde{u}$ over element $e$ is $\tilde{u}^{e}$. The source residual is $r=\nabla^{2} \tilde{u}+f$ in each $\Omega^{e}$ and is conventionally zero over element sides. For each element $e$ we denote the boundary-normal flux derived from $\tilde{u}$ as $q^{u e}=k \partial \tilde{u}^{e} / \partial \mathbf{n}^{e}$ where $\mathbf{n}^{e}$ is the unit outward normal on $\Gamma^{e}$. On each internal side $\Gamma^{e d}$ shared by elements $e$ and $d$ we select a common normal direction $\mathbf{n}^{\text {ed }}$ and define the flux jump as

$$
\llbracket q^{u e d} \rrbracket=q^{u e} \cos \left(\mathbf{n}^{e}, \mathbf{n}^{d e}\right)-q^{u d} \cos \left(\mathbf{n}^{d}, \mathbf{n}^{d e}\right),
$$

a value that is independent of the choice for $\mathbf{n}^{e d}$. Let $w=w(x, y) \in H^{1}(\Omega):\left.w\right|_{\Gamma_{d}}=0$ be a test function. Introduce the usual bilinear form associated with the internal energy (14)

$$
U(u, w)=\int_{\Omega}(\operatorname{div} u)^{T} \operatorname{div} w d \Omega .
$$

On integrating by parts one finds that the error satisfies

$$
\begin{aligned}
U(u-\tilde{u}, w)=U\left(\epsilon^{u}, w\right) & =\int_{\Omega} f w d \Omega+\sum_{e=1}^{N_{e}} \int_{\Omega^{e}} \nabla^{2} \tilde{u} w d \Omega+\int_{\Gamma_{q}} \widehat{q} w d \Gamma-\sum_{e=1}^{N_{e}} \int_{\Gamma^{e}} \frac{\partial \tilde{u}}{\partial \mathbf{n}_{e}} w d \Gamma \\
& =\int_{\Omega} r w d \Omega+\int_{\Gamma_{q}}\left(\widehat{q}-q^{u}\right) w d \Gamma-\sum_{e, d} \int_{\Gamma^{e d} \in \Gamma_{i}} \llbracket q^{u e d} \rrbracket w d \Gamma,
\end{aligned}
$$

which may be called the Weak Form of the Error (WFE). It displays three contributions. The first term shows the effect of the source-residual error $r=\nabla^{2} \tilde{u}+f$ over each element. The second term brings the effect of violating the flux boundary conditions. The third term gives the contribution of interelement flux jumps.

The error energy measure is obtained by taking $w=u-\tilde{u}=\epsilon^{u}$ :

$$
R \stackrel{\text { def }}{=} U\left(\epsilon^{u}, \epsilon^{u}\right)=\int_{\Omega} r \epsilon^{u} d \Omega+\int_{\Gamma_{q}}\left(\widehat{q}-q^{u}\right) \epsilon^{u} d \Gamma-\sum_{e, d} \int_{\Gamma^{e d} \in \Gamma_{i}} \llbracket q^{u e d} \rrbracket \epsilon^{u} d \Gamma
$$

which may be called the Error Energy Equation (EEE). Relations of this nature are the basis for developing a posteriori local error estimators for the Laplace and Poisson equations as well as (with suitable extensions) the Stokes and elasticity problems. There is an abundant and rapidly growing literature in this topic. The state of the art is typified by the research of Oden and coworkers $[16,48,49]$.

Several technical difficulties, however, may be noted.

1. Both WFE and EEE involve the exact solution $u$, which is of course unknown. Consequently a higher order estimation is inevitable. One way to achieve this is by 


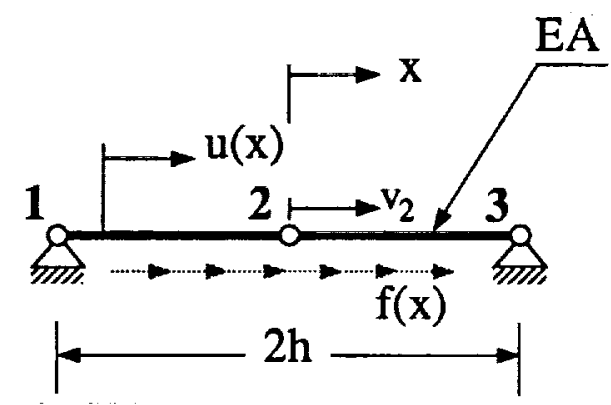

Fig. 17. Bar problem used to illustrate behavior of HOE error indicator.

considering element patches (macroelements) and smoothing $\tilde{u}$, as in the widely used projection method of Zienkiewicz and Zhu [67]. Another group of techniques relies on hierarchical approximations $[64,65,66]$.

2. Many interesting problems display jumps (e.g. at shocks, wavefronts, plastic bands) in the exact solution. The resulting lack of smoothness - in the previous case, $u$ can be no longer assumed in $H^{\mathrm{I}}(\Omega)$ - requires substantial revisions in the analysis.

3. The FE solution has been assumed to be variationally consistent with Eq. (14), that is, fully conforming. But in many practical problems, especially in structural and continuum mechanics applications, interelement continuity is lost. This may be due to several factors. First, as noted in Section 10 most high-performance finite elements (HPFEs) abandon conformity from the outset. Second, the treatment of complicated 3-D structures such as shells, folded plates or stiffened panels often introduces discontinuities even if the constituent elements (by miracle) were fully conforming.

4. Although a WFE such as (73) can be formally extended without difficulty to PVPs thus fitting HPFEs, the resulting EEE is generally indefinite and thus unsuitable as an error measure. It is preferable to keep error measures positive definite, as in the example that follows, by concentrating on a single field of interest to the user.

\subsection{The 3-Node Bar Element Revisited}

To motivate the approach discussed below, it is useful to consider again the 3-node bar element of Figure 11, now as the problem depicted in Figure 17. This example connects to the Poisson's equation discussed above by simply taking $k=E A, u$ as axial displacement, and $f$ as prescribed axial force. Domains $\Omega$ and $\Gamma$ reduce to $x$-aligned segments and end node points, respectively. The bar ends are assumed fixed: $v_{1}=v_{3}=0$; therefore $\check{v}_{2}=v_{2}$. End fixity is not an important restriction because any element must exactly capture the linear motion $u_{b}$ of (64).

We investigate here an assumed strain element variant of that considered in Section 10.2. It is based on the ANDES functional (already mentioned in Section 10.4) that is obtained by taking $j_{11}=\alpha-1, j_{22}=\alpha, j_{33}=0$, and which reduces to HW for $\alpha=1$ and to HR for $\alpha=0$. The assumptions on $\tilde{N}, \tilde{e}$ and $\tilde{d}$ are as before. The displacement field is again 
$u=u_{b}+u_{h}$ but its higher order part is now

$$
u_{h}=v_{2}\left[\frac{\mu x}{h} \operatorname{sign}(x)-(1-\mu) \frac{x^{2}}{h^{2}}+1\right]
$$

rather than (64). This is obtained by integrating the assumed strain (65).

The applied force is $f(x)=\bar{f}$, a constant. The finite element matrix equations reduce to the scalar equation $k_{22} v_{2}=p_{2}$, in which

$$
p_{2}=\frac{(4-\mu)}{3} h \bar{f}, \quad k_{22}=\frac{2 E A \beta}{h}, \quad \beta=\frac{1}{3} \alpha\left(4-2 \mu+\mu^{2}\right), \quad v_{2}=\frac{4-\mu}{2 \alpha\left(4-2 \mu+\mu^{2}\right)} \frac{h^{2} \bar{f}}{E A} .
$$

The error energy equation is derived from the computed strains:

$$
R=\frac{1}{2} \int_{-h}^{h} E A[e(x)-\tilde{e}(x)]^{2} d x=\frac{8(1-\alpha)^{2}(2-\mu)+\left(1-2 \alpha+4 \alpha^{2}\right) \mu^{2}}{12 \alpha^{2}\left(4-2 \mu+\mu^{2}\right)} \frac{h^{3} \bar{f}^{2}}{E A},
$$

[Using $e^{u}$ yields the same result here because $\tilde{e} \equiv e^{u}$ on account of (75). Note that only if $\alpha=1$ and $\mu=0$ the element yields the exact solution for this problem.] Now comparing (77) to the higher order energy (HOE) absorbed by the element

$$
U_{h}=\frac{1}{2} \mathbf{v}^{T} \mathbf{K}_{h} \mathbf{v}=\frac{1}{2} k_{22} v_{2}^{2}=\frac{1}{2} p_{2} v_{2}=\frac{(4-\mu)^{2}}{\alpha\left(4-2 \mu+\mu^{2}\right)} \frac{h^{3} \bar{f}^{2}}{E A}
$$

we note the same dependence on problem properties $E A, h$ and $\bar{f}$. The error coefficients of $R$ and $U_{h}$ match if we choose

$$
\begin{aligned}
& \alpha=\frac{48-24 \mu+3 \mu^{2} \pm(4-\mu) \sqrt{80-40 \mu-7 \mu^{2}}}{8\left(4-2 \mu+\mu^{2}\right)} \text { (given } \mu \text { ), } \\
& \mu=\frac{4-12 \alpha+4 \alpha^{2} \pm 4 \sqrt{3} \alpha \sqrt{3 \alpha-\alpha^{2}-1}}{1-3 \alpha+4 \alpha^{2}} \quad \text { (given } \alpha \text { ). }
\end{aligned}
$$

A particularly interesting combination is $\alpha=1, \mu=2 \sqrt{3}-2$. If one departs from these $(\alpha, \mu)$ pairs, $U_{h}$ and $R$ do not display the same error coefficients. In the accepted FEM terminology, (78) would be an error indicator but not an estimator. Of course, should one select $\alpha$ and $\mu$ beforehand, matching is always possible by choosing $\beta$ differently for the higher order stiffness and error energy computations. This matching game can be played with other functionals and displacement-strain assumptions.

The example is instructive and may be extended to more general force distributions, geometry and boundary condition data, as well as to $C^{1}$ and $C^{0}$ beams. But of course one-dimensional problems are of little interest in practice. Can the underlying idea of "higher error energy" be useful as an indicator or even estimator for more general situations? The PVP framework provides the tools to at least pose this problem. 


\subsection{Local Error Estimation Using IPVPs}

Consider again the IPVP of classical elasticity with in which three free parameters are generically shown as $\alpha, \beta$ and $\gamma$ in the argument list:

$$
\Pi(\tilde{\mathbf{u}}, \tilde{\boldsymbol{\sigma}}, \tilde{\mathbf{e}} ; \alpha, \beta, \gamma)=U(\tilde{\mathbf{u}}, \tilde{\boldsymbol{\sigma}}, \tilde{\mathbf{e}} ; \alpha, \beta, \gamma)-P(\tilde{\mathbf{u}}, \tilde{\boldsymbol{\sigma}}, \tilde{\mathbf{e}}),
$$

Here $P$ stands for either $P^{c}, P^{d}$ or $P^{t}$. If not $P^{c}$ an additional boundary field $\tilde{\mathrm{d}}$ or $\tilde{\mathrm{t}}$ would appear in the argument list for $P$. This is of no consequence to the argument that follows because the potential $P$ is not affected by the parametrization.

Now suppose that (80) is used to construct a finite element discretization, while keeping one or more parameters free. Practically this means that such parameters are kept as arguments of the element stiffness formation subroutines. The discrete approximants $\tilde{\mathbf{u}}(\alpha, \beta, \gamma), \tilde{\boldsymbol{\sigma}}(\alpha, \beta, \gamma)$ and $\tilde{\mathbf{e}}(\alpha, \beta, \gamma)$ obtained on a fixed mesh can be viewed as functions of the free parameters.

Because (80) is a IPVP, $U$ takes the same value if the varied fields $\tilde{\mathbf{u}}, \tilde{\boldsymbol{\sigma}}$, $\tilde{\mathbf{e}}$ were set to the exact solution fields $\mathbf{u}, \boldsymbol{\sigma}, \boldsymbol{\epsilon}$, independently of $\alpha, \beta$ and $\gamma$. Hence, we may expect that the difference between two values of $U$ obtained for two different sets of parameters may provide a measure on how far we are from the converged solution. That is, the difference may be adopted as an error indicator. The most interesting feature of such an indicator is that it naturally provides an element level measure, as the following argument shows.

To tackle the general case first, suppose that the two sets of parameters are $\left(\alpha_{1}, \beta_{1}\right.$, $\left.\gamma_{1}\right)$ and $\left(\alpha_{0}, \beta_{0}, \gamma_{0}\right)$. The corresponding approximate values for displacements, strains and stresses obtained with a given mesh are $\left(\tilde{\mathbf{u}}_{1}, \tilde{\boldsymbol{\sigma}}_{1}, \tilde{\mathbf{e}}_{1}\right)$ and $\left(\tilde{\mathbf{u}}_{0}, \tilde{\boldsymbol{\sigma}}_{0}, \tilde{\mathbf{e}}_{0}\right)$, respectively. Denote by $U_{1}^{e}=U^{e}\left(\tilde{\mathbf{u}}_{1}, \tilde{\boldsymbol{\sigma}}_{1}, \tilde{\mathbf{e}}_{1}, \alpha_{1}, \beta_{1}, \gamma_{1}\right)$ and $U_{0}^{e}=U^{e}\left(\tilde{\mathbf{u}}_{0}, \tilde{\boldsymbol{\sigma}}_{0}, \tilde{\mathbf{e}}_{0}, \alpha_{0}, \beta_{0}, \gamma_{0}\right)$ the value of the generalized strain energy evaluated over the $e^{\text {th }}$ element of that mesh. Then the element error indicator is defined as the difference

$$
R^{e}=\left|U_{1}^{e}-U_{0}^{e}\right| \text {. }
$$

The definition (81) apparently requires that the problem be solved twice for a given mesh. Additional assumptions and manipulations discussed by Beltrán [8], Militello [42] and Militello and Felippa [45] show that the error indicator (81) can be expressed in the onesolution form

$$
U_{h}^{e}=\frac{1}{2}\left(\mathbf{v}^{e}\right)^{T} \mathbf{K}_{h}^{e} \mathbf{v}^{e}
$$

where $\mathbf{v}^{e}$ denotes the element node displacement vector extracted from the complete discrete solution for a single parameter set $(\alpha, \beta, \gamma)$. Because only one solution is involved, we shall refer to this as a one-solution indicator. Physically (82) is the higher order energy (HOE) absorbed by the element and already encountered in Section 11.2.

Inasmuch as for the HP elements discussed in Section 10 the matrices of the decomposition (62) are usually available in separate form, this indicator can be readily computed in an element-by-element manner. No calculation of interelement stress-flux jumps is necessary.

It should be observed that the indicator (82) is related to that heuristically proposed by Melosh and Marcal [39] in the context of the SED (Strain Energy Density) method. 


\subsection{Historical Note and Discussion}

Certainly (82) is computationally attractive, as in principle it eliminates all of the technical objections raised in Section 11.1 against the "residual plus jumps" estimators. It is also physically transparent because it "filters out" contributions from the rigid body and constant stress states. Thus, in problems whose analytical solution consist of uniform stress states the indicator vanishes over each element, in accordance with the fact that any mesh of complete elements should solve those cases exactly. This statement may be extended to problems solved by constant stress states that jump at internal interfaces, if such interfaces are modeled correctly by the FE mesh.

The key question is: can $U_{h}^{e}$ be considered an error estimator? Little theory is available to answer this question, aside from rather trivial one-dimensional cases such as that discussed in Section 11.2. Evidence to date is based largely on numerical experiments.

The first experiments were conducted by Beltrán [8] and presented by Beltrán and Alarcón $[9,10,11]$. They exploited the Scaled Free Formulation functional $\Pi_{\gamma}$ to derive a $\mathrm{HOE}$ error indicator for plane stress and plate bending problems. This indicator was applied to $h$ (element subdivision) and $p$ (element enrichment) mesh adaptation processes. It was concluded that performance was generally satisfactory although connection with the actual error was erratic.

Militello [42] conducted experiments in plate bending and shell problems with elements formulated with the Assumed Natural Deviatoric Strain (ANDES) formulation. The indicator was applied to both $r$ (node relocation) and $r h$ mesh adaptation processes. The general conclusion is that the indicator was effective in driving those processes in flat plates. In shells the performance was less consistent possibly because (82) cannot account for geometric discretization error without information from adjacent elements.

Most experiments so far have dealt with fairly simple elements in which the higher-order stiffness is an incomplete-polynomial correction to the basic behavior. This is in agreement with the philosophy that HP elements should be "simple but not simplex." If the HOE indicator is to be extended to more refined elements, the appropriate decomposition of the element stiffness matrix is not $\mathbf{K}^{e}=\mathbf{K}_{b}^{e}+\mathbf{K}_{h}^{e}$ but

$$
\mathbf{K}^{e}=\mathbf{K}_{b}^{e}+\mathbf{K}_{h c}^{e}+\mathbf{K}_{h h}^{e},
$$

where the complete higher order stiffness $\mathbf{K}_{h c}^{e}$ is produced by displacement-stress-strain expansions complete up to a certain polynomial order, and the hierarchical higher order stiffness is due to additional terms. The HOE indicator is then $\frac{1}{2}\left(\mathbf{v}^{e}\right)^{T} \mathbf{K}_{h h}^{e} \mathbf{v}^{e}$. Such refined elements appear in $p$ mesh-adaptation processes.

The connection of such indicators to hierarchical error estimators is an open research topic. From evidence gathered to date it appears that free parameters for HOE evaluation need to be adjusted from those used for the stiffness calculations - as in the example of Section 11.2 - so as to get an error estimator rather than just an indicator. In any case, it is obvious that if $\mathbf{K}_{h k}$ vanishes the HOE indicator is vacuous. Thus complete elements such 
as the Linear Strain Triangle or Quadratic Strain Triangle [17] should be viewed as useless for this purpose.

\section{APPLICATION 3: FINITE ELEMENT TEMPLATES}

The following stiffness matrix decomposition has been observed to recur in several FF and ANDES elements (in particular, those mentioned in Section 10):

$$
\mathbf{K}=\mathbf{K}_{b}+\mathbf{K}_{h}=c \mathbf{L E L}^{T}+\beta \mathbf{T}_{h}^{T} \mathbf{S} \mathbf{T}_{h},
$$

where $c$ is the inverse of the element volume, area or length, $\beta$ is a positive scaling factor, $\mathbf{L}$ and $\mathbf{T}_{h}$ are geometric matrices, $\mathbf{E}$ is the matrix of elastic moduli, and $\mathbf{S}$ depends on the geometry and constitutive behavior as well as variational principle parametrization. (The element index $e$ has again been omitted from (84) for simplicity.)

Additional properties are as follows. The geometric matrix $\mathrm{L}$, which is called the forcelumping matrix in accordance with the FF terminology [12], is responsible for satisfaction of the patch test as well as that of element mixability properties; a detailed discussion of these points has been recently given by Militello and Felippa [44]. The geometric matrix $\mathbf{T}_{h}$ annihilates the rigid-body modes and constant strain states. Together with $\mathbf{S}$ it provides the correct rank to $\mathbf{K}_{h}$, and thus is responsible for stability and accuracy.

Both $\mathbf{L}$ and $\mathbf{S}$ may contain free parameters. Any free parameters in $\mathbf{L}$, however, must be the same for all elements in an assembly; otherwise convergence and mixability are impaired. On the other hand, parameters in $\mathbf{S}$, as well as $\beta$, may vary from element to element as long as the correct rank is preserved.

The form (84) will be called a finite element template, or template for short. The template conjecture asserts that (84) is the most general form for all convergent $F E$ of given degreeof-freedom configuration.

A parametrized expression that provides all such elements is called an universal template. A parametrized expression that provides a practically important subset of all such elements is called a generic template. Equations (69) and (70) provide examples of universal templates for the 3-node bar and 2-node plane beam element, respectively.

Verification of this conjecture for more general elements would have the following projected implications:

1. The confusion associated with the enormous number of "named" elements claimed in the literature clears up: such elements, just like the canonical functionals discussed previously, become points in a parametric continuum. Among other benefits, such unification should help to streamline teaching of finite element theory and hence release instruction time to cover problem modeling aspects.

2. Construction and testing of parametrized FE libraries can be greatly simplified.

3. Another realm of mesh adaptivity opens up: $c$ adaptation, in which programs can pick up elements from the parametrized continuum to strive for maximum accuracy on a fixed mesh. 
4. Several persistent mysteries may be cleared once and for all: convergence, mixability, accuracy, locking, spurious modes, and distortion sensitivity. These properties could be traced to components of the template (84) and interpreted physically.

\section{CONCLUSION}

The paper has surveyed the development of Parametrized Variational Principles and selected applications to the Finite Element Method. It is shown that multifield PVPs provide a framework by which classical variational principles may be embedded into a parametrizable continuum of functionals.

Three FEM applications have been reviewed: construction of high performance finite elements, local error estimation, and element templates. These are in different stages of development. While the application to HPFEs has succeeded beyond initial expectations, the development of element-level error estimation has primarily relied on numerical experiments, and the notion of templates is largely a conjecture suggested by isolated examples.

In the author's view, the following areas of variational methods merit further development:

- Parametrization of boundary terms, and use of such functionals in interfacing finite element and variational Boundary Element discretizations (or, more generally, Trefftz methods).

- Extension to dynamic and nonlinear problems. It is well known that finite elements optimized for linear static analysis may not perform as well when time-dependency and nonlinear effects are included. Functional parametrization opens up possibilities of extending the aforementioned FEM applications to such cases.

The following FEM-application areas would benefit from focused research work:

- Establishment of a general convergence theory of FEM based on PVPs. Areas that require special attention are: dependence of element performance on choice of parameters, mixability properties, and limitation principles.

- Development of a sound a-posteriori error estimation methodology for two- and threedimensional nonconforming elements; in particular establishing connections between HOE and hierarchical error estimators.

- Verification or disproval of the template conjecture. If proven, proceed to exploit the benefits outlined in Section 12.

\section{Acknowledgements}

The author is indebted to Professor Bernard Nayroles for the invitation to address the 1992 SIMG. The preparation of this work has been supported by NASA Langley Research Center under Grant NAS1-756, monitored by Dr. J. Housner. 


\section{References}

1. D. J. Allman, A compatible triangular element including vertex rotations for plane elasticity analysis, Computers \& Stmetures, 19 (1984) 1-8

2. K. Alvin, H. M. de la Fuente, B. Haugen and C. A. Felippa, Membrane elements with corner drilling freedoms: I. The EFF element, Finite Elements in Analysis and Design, 12, (1992), 163-187

3. J. H. Argyris and S. Kelsey, Energy Theorems and Structural Analysis, Butterworths Sci. Pubs., London (1960); reprinted from Aircraft Engrg. 26 (1954) and 27 (1955)

4. I. Babuška, The selfadaptive approach in the finite element method, in The Mathematics of Finite Elements and Applications II MAFELAP 75, ed. by J. R. Whiteman, Academic Press, London (1975) 125-142.

5. I. Babuška and A. Miller, A feedback finite element method with a-posteriori error estimation: Part I. The finite element method and some basic properties of the a-posteriori error indicator, Comput. Methods Appl. Mech. Engrg., 61 (1987) 1-40

6. I. Babuška, R. Durán and R. Rodriguez, Analysis of the efficiency of an a-posteriori error estimator for linear triangular finite elements, SIAM J. Numer. Anal., 29 (1992) 947-964

7. K. J. Bathe and E. N. Dvorkin, A four-node plate bending element based on Mindlin/Reissner plate theory and a mixed interpolation, Int. J. Numer. Meth. Engrg., 21 (1985) 367-383

8. F. J. Beltrán, Estimadores de error basados en principios variacionales multicampo para cálculos por elementos finitos, Tesis Doctoral, Universidad Politécnica de Madrid (1990)

9. F. J. Beltrán and E. Alarcón, Estimadores del error de discretización con principios variacionales multicampo: I. Elasticidad, Rev. Internat. Métodos Numer. Cálculo y Diseño en Ingenieria, 8 (1992) 43-55

10. F. J. Beltrán and E. Alarcón, Estimadores del error de discretizaci'on con principios variacionales multicampo: II. Flexión de placas, Rev. Internat. Métodos Numer. Cálculo y Diseño en Ingenieria, 8 (1992) 139-156

11. F. J. Beltrán and E. Alarcón, Accuracy estimates based on multifield variational principles, Eur. J. Mech. A/Solids, 11 (1992) 487-518

12. P. G. Bergan and M. K. Nygård, Finite elements with increased freedom in choosing shape functions, Internat. J. of Numerical Methods in Engineering, 20 (1984) 643-664

13. P. G. Bergan and C. A. Felippa, A triangular membrane element with rotational degrees of freedom, Comput. Methods Appl. Mech. Engrg., 50 (1985) 25-69

14. R. W. Clough, The finite element method in plane stress analysis, J. Struct. Div. ASCE, Proc. 2nd Conf. Electronic Computation (1961) 345-378

15. R. Courant, Variational methods for the solution of problems of equilibrium and vibrations, Bull. Amer. Math. Soc., 49 (1943) 1-23

16. L. Demkowicz, J. T. Oden, W. Rachowicz and O. Hardy, Towards a universal $h$ - $p$ adaptive finite element strategy, Part 1. Constrained approximation and data structure, Comput. Methods Appl. Mech. Engrg., 77 (1989) 79-112

17. C. A. Felippa, Refined finite element analysis of linear and nonlinear two-dimensional structures, Ph. D. Dissertation, Department of Civil Engineering, University of California at Berkeley, Berkeley, CA (1966) 
18. C. A. Felippa and P. G. Bergan, A triangular plate bending element based on an energyorthogonal free formulation, Comput. Methods Appl. Mech. Engrg., 61 (1987) 129-160

19. C. A. Felippa, Parametrized multifield variational principles in elasticity: I. Mixed functionals, Commun. Appl. Numer. Methods, 5 (1989) 69-78

20. C. A. Felippa, Parametrized multifield variational principles in elasticity: II. Hybrid functionals and the free formulation, Commun. Appl. Numer. Methods, 5 (1989) 79-88

21. C. A. Felippa, The extended free formulation of finite elements in linear elasticity, J. Appl. Mech., 56 (1989) 609-616

22. C. A. Felippa and C. Militello, Developments in variational methods for high performance plate and shell elements, in Analytical and Computational Models for Shells, CED Vol. 3, ed. by A. K. Noor, T. Belytschko and J. C. Simo, The American Society of Mechanical Engineers, ASME, New York (1989) 191-216

23. C. A. Felippa and C. Militello, Variational Formulation of High Performance Finite Elements: Parametrized Variational Principles, Computers \& Structures, 36 (1990) 1-11

24. C. A. Felippa, Parametrized variational principles encompassing compressible and incompressible elasticity, Internat. J. Solids Structures, 29 (1991) 57-68

25. C. A. Felippa, Parametrized variational principles for micropolar elasticity, Internat. J. Solids Structures, 29 (1992) 2709-2721

26. C. A. Felippa and C. Militello, Membrane elements with corner drilling freedoms: II. The ANDES element, Finite Elements in Analysis and Design, 12 (1992) 189-201

27. C. A. Felippa and Alexander, S., Membrane elements with corner drilling freedoms: III. Implementation and performance validation, Finite Elements in Analysis and Design, 12 (1992) 203-239

28. C. A. Felippa and J. Schuler, Parametrized variational principles for electromagnetodynamics, Proceeding ASME/ASCE Conference Charlottesville, VA June 1993, to appear in bound ASME volume

29. R. A. Frazer, W. J. Duncan and A. R. Collar, Elementary Matrices, Cambridge Univ. Press, (1963) (First edition 1938)

30. C. Fox, An Introduction to the Calculus of Variations, Dover, New York (1950)

31. F. Hartmann, The Mathematical Basis of Structural Mechanics, Springer-Verlag, Berlin, 1985

32. H. C. Huang and E. Hinton, A new nine node degenerated shell element with enhanced membrane and shear interpolation, Int. J. Numer. Meth. Engrg., 22 (1986) 73-92

33. T. J. R. Hughes and F. Brezzi, On drilling degrees of freedom, Comput. Methods Appl. Mech. Engrg., 72 (1989) 105-121

34. B. Langefors, Analysis of elastic structures by matrix coefficients, with special regard to semimonocoque structures, J. Aero. Sci., 19 (1952) 451-458

35. S. Levy, Computation of influence coefficients for aircraft structures with discontinuities and sweepback, J. Aero. Sci., 14, (1947) 547-560

36. S. Levy, Structural analysis and influence coefficients for delta wings, J. Aero. Sci., 20, (1953) 677-684

37. S. Liu and Z. Jiang, General form of the generalized variational principle in elasticity, Commun. Appl. Numer. Methods, 5 (1988) 749-760 
38. S. Liu, Extended Gurtin's variational principles for linear elastodynamics, in Computational Mechanics '91, ed. by S. N. Atluri, D. E. Beskos, R. Jones and G. Yagawa, ICES Publications, Atlanta, GA (1991) 649-650

39. R. J. Melosh and P. V. Marcal, An energy basis for mesh refinement of structural continua, Internat. J. Numer. Methods Engrg., 11 (1977) 1083-1091.

40. C. Militello and C. A. Felippa, A variational justification of the assumed natural strain formulation of finite elements: I. Variational principles, Computers \& Structures, 34 (1990) $431-438$

41. C. Militello and C. A. Felippa, A variational justification of the assumed natural strain formulation of finite elements: II. The four-node $C^{0}$ plate element, Computers $\&$ Structures, 34 (1990) 439-444

42. C. Militello, Application of Parametrized Variational Principles to the Finite Element Method, Ph. D. Dissertation, Department of Aerospace Engineering Sciences, University of Colorado, Boulder, CO (1991)

43. C. Militello and C. A. Felippa, The first ANDES elements: 9-dof plate bending triangles, Comput. Methods Appl. Mech. Engrg., 93 (1991) 217-246.

44. C. Militello and C. A. Felippa, The individual element test revisited, in The Finite Element Method in the 1990s, ed. by E. Oñate, J. Periaux and A. Samuelsson, Springer-Verlag, Berlin (1991) 554-564

45. C. Militello and C. A. Felippa, $r$-adaptive methods based on element level error estimators for parallel analysis of plates and shells, presented to First US National Congress on Computational Mechanics, Chicago (July 1991); also in 33rd AIAA/ASCE/ASME/AIH Structures, Structural Dynamics and Materials Conference Proceedings, Dallas (April 1992)

46. J. T. Oden and J. N. Reddy, On dual complementary variational principles in mathematical physics, Int. J. Engrg. Sci., 12 (1974) 1-29

47. J. T. Oden and J. N. Reddy, Variational Methods in Theoretical Mechanics, Springer-Verlag (1982)

48. J. T. Oden and L. Demkowicz, Advances in adaptive improvements: A survey of adaptive methods in computational mechanics, in State of the Art Surveys in Computational Mechanics, ed. by A. K. Noor and J. T. Oden, ASME, New York (1988) 441-468

49. J. T. Oden, L. Demkowicz, W. Rachowicz and T. A. Westermann, Towards a universal $h-p$ adaptive finite element strategy, Part 2. A posteriory error adaptation, Comput. Methods Appl. Mech. Engrg., 77 (1989) 113-180

50. K. C. Park and G. M. Stanley, A curved $C^{0}$ shell element based on assumed naturalcoordinate strains, J. Appl. Mech., 53 (1976) 278-290

51. T. H. H. Pian and P. Tong, Basis of finite element methods for solid continua, Internat. J. Numer. Methods Engrg., 1 (1969) 3-28

52. T. H. H. Pian, Finite element methods by variational principles with relaxed continuity requirements, in Variational Methods in Engineering, Vol. 1, ed. by C. A. Brebbia and H. Tottenham, Southampton University Press, Southhampton (1973)

53. J. N. Reddy, On complemnentary variational principles for the linear theory of plates, Joumal of Structural Mechanics, 4 (1976) 417-436

54. J. N. Reddy, Energy and Variational Methods in Applied Mechanics, Wiley/Interscience, New York, 1984 
55. R. Santilli, Foundations of Theoretical Mechanics I, Springer-Verlag, Berlin (1978)

56. G. Sewell, An adaptive computer program for the solution of $\operatorname{Div}(P(X, Y) \operatorname{Grad} U)=$ $F(X, Y, U)$ on a polynomial region, in The Mathematics of Finite Elements and Applications II MAFELAP 75, ed. by J. R. Whiteman, Academic Press, London (1975) 543-554.

57. M. J. Sewell, Maximum and Minimum Principles, Cambridge, (1987)

58. J. C. Simo and T. J. R. Hughes, On the variational foundations of assumed strain methods, J. Appl. Mech., 53 (1986) 51-54

59. E. Tonti, On the mathematical structure of a large class of physical theories, Accad. Naz. dei Lincei, Series III, 52 (1972) 48-56

60. E. Tonti, A mathematical model for physical theories, Accad. Naz. dei Lincei, Series VIII, 52 (1972) 175-181, 350-356

61. M. J. Turner, R. W. Clough, H. C. Martin and L. J. Topp, Stiffness and deflection analysis of complex structures, J. Aero. Sci., 23 (1956) 805-823

62. B. D. Vujanovic and S. E. Jones, Variational Methods in Nonconservative Phenomena, Academic Press (1989)

63. K. Washizu, Variational Methods in Elasticity and Plasticity, Pergamon Press (1972)

64. O. C. Zienkiewicz, D. W. Kelly, J. P. Gago, J. P. and I. Babuška, Hierarchical finite element approaches, error estimates and adaptive refinement, in The Mathematics of Finite Elements and Applications IV (MAFELAP VI), ed. by J.R. Whiteman, Academic Press, New York (1981)

65. O. C. Zienkiewicz and A. Craig, A-posteriori error estimates and adaptive mesh refinement in the finite element method concepts, Chapter 5 in The Mathematical Basis of Finite Element Methods, ed. by D. Griffiths, Clarendon Press, Oxford (1984)

66. O. C. Zienkiewicz and A. Craig, Adaptive refinement, error estimates, multigrid solution and hierarchic finite element method concepts, Chapter 2 in Accuracy Estimates and Adaptive Refinements in Finite Element Computations, ed. by I. Babuška et.al., John Wiley and Sons, Chichester (1986)

67. O. C. Zienkiewicz and J. Z. Zhu, A simple error estimator and adaptive procedure for practical engineering analysis, Internat. J. Numer. Methods Engrg., 24 (1987) 337-357. 


\section{COMPUTER METHODS IN APPLIED MECHANICS AND ENGINEERING}

Editors:

John. H. Argyris

Thomas J.R. Hughes

J. Tinsley Oden
Institute for Computer Applications, - Editorial Office -

Pfaffenwaldring 27, W-7000 Stutrgart 80, Germany, Tel. No.: (07II) 685-3593

Imperial College of Science and Technology, Prince Consori Rd., London SW7, United Kingdom

Stanford University, Division of Applied Mechanics, Durand Building, Stanford, California 94305-4040, USA, Tel. No.: (415) 723-2040, Fax No.: (415) 723-1778

TICOM Office, 3500 West Batcones Center Drive, Austin, Texas 78759, USA, Tel. No.: (5I2) 47I-3312, Fax No.: (512) 471-8694

April 5, 1993

Dr. Carlos Felippa

University of Colorado

Department of Aerospace Engineering and

Center for Space Structures and Controls

Boulder, CO 80309

Dear Carlos:

Ref: \#404 "Parametrized Variational Principles and Applications To Computational Mechanics"

by Carlos Felippa

We have now obtained a review on your paper, "Parametrized Variational Principles and Applications To Computational Mechanics". The reviewers state that this is an excellent paper, although it requires a few minor revisions. Please look over the enclosed comments and, if you wish, submit a revised version of the paper for a quick final review.

Thank you for allowing Computer Methods in Applied Mechanics and Engineering to consider your work, and we look forward to hearing from you.

Sincerely,

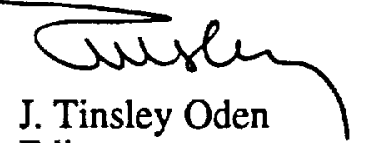

Editor

JTO/c

Enclosure 